\title{
Photosynthetic activity and productivity before and after ear emergence in spring wheat
}

\author{
H. BIRECKA, L. DAKIĆ-WEODKOWSKA
}

In continuation of previous investigations on spring wheat (Birecka, Dakić-Włodkowska 1963, 1964) main attention was paid in the present experiments to 1) changes with age in the contribution of the particular green parts to the total plant photosynthetic activity beginning from the $43-47^{\text {th }}$ day of growth; 2 ) redistribution of assimilates during the period from their formation to ripeness and 3) influence of a higher nitrogen supply (at shooting) on the plant photosynthetic activity as well as on the final accumulation of assimilates.

Two awned wheat varieties were investigated: Chłopicka and Popularna.

\section{METHODS}

Experiment 1963. Full sprouting of plants sown in pots filled with $8 \mathrm{~kg}$ of a mixture of soil and sand - with $\mathrm{N}-0.4, \mathrm{P}_{2} \mathrm{O}_{5}-0.3$ and $\mathrm{K}_{2} \mathrm{O}-0.5 \mathrm{~g}$ added - was noted on April 24, tillering $12-13$ days later. At that time the plants were given additionally $\mathrm{N}, \mathrm{P}_{2} \mathrm{O}_{5}$ and $\mathrm{K}_{2} \mathrm{O}-0.3 \mathrm{~g}$ of each per pot. They were thinned gradually from 18 to 12 . Shooting in both varieties was noted on the $33^{\text {rd }}$ day of growth. Two days later the plants of each variety were divided into two groups; the first was given 0.2 , the second $0.6 \mathrm{~g} \mathrm{~N}$ per pot. Heading of the main shoot was noted in Chłopicka on the $51^{\text {st }}$, in Popularna $-52-53^{\text {rd }}$ day after sprouting, flowering $5-6$ days later. Heading of tillers in both varieties was observed on the $60-63^{\text {rd }}$ day. Soil humidity was maintained at $60 \%$ of its water holding capacity.

The plants were sampled for dry matter determination 6 times (first sampling on the $43^{\text {rd }}$ day of growth) at various intervals till ripeness. In the middle of each period between samplings (the last one excepted) groups of plants representing both varieties at two $\mathrm{N}$ levels were exposed in a plexiglass box (out-of-doors) at 10 a.m. to ${ }^{14} \mathrm{C}$-labelled $\mathrm{CO}_{2}$ for $20-30 \mathrm{~min}$ then they were removed and placed under normal conditions (during daytime - under a wire net, at night under glass). They were sampled immediately after exposure, 20 hours later, on the day of the next two samplings for dry matter determination and at ripeness (plants at high $\mathrm{N}$ level were sampled only twice, at the first and last harvest). On the day of exposure chlorophyll content in the particular green parts as well as the lamina area was determined. 
Experiment 1964. Seeds of Popularna were sown on April 15, and those of Chłopicka two days later. Full sprouting was noted on April 20 and 22, respectively. Mineral nutrients added till shooting were as follows: $\mathrm{N}-0.8, \mathrm{P}_{2} \mathrm{O}_{5}-0.8$, $\mathrm{K}_{2} \mathrm{O}-1.2 \mathrm{~g}$ per pot.

Shooting was observed on May 24; at that time the plants were thinned to 9 . Two days later one group was given 02 , the second $-0.7 \mathrm{~g} \mathrm{~N}$ per pot. Full ear emergence on the main shoot of both varieties was observed on the $53^{\text {rd }}$ day of growth and that of the first tiller three or four days later.

The first sampling was performed on the $39^{\text {th }}$ day after sprouting and repeated at various intervals. In the middle of each interval the plants were exposed to ${ }^{14} \mathrm{CO}_{2}$. The technical procedures applied were similar as in expt. 1963 (but no samples were taken 20 hours after exposure). In table 1 the conditions, under which the plants were kept in the box, are listed.

\section{Table 1}

Conditions, under which plants were kept in plexiglass box

\begin{tabular}{|c|c|c|c|c|c|c|}
\hline No. & $\begin{array}{c}\text { Age } \\
\text { of plants } \\
\text { days }\end{array}$ & $\begin{array}{c}\mathrm{CO}_{2} \text { content } \\
\text { vol. } \%\end{array}$ & $\begin{array}{c}\text { Radioactivity } \\
\text { applied } \\
\text { mc }\end{array}$ & $\begin{array}{l}{ }^{14} \mathrm{CO}_{2} \text { assi- } \\
\text { milated }-\% \\
\text { of total } \\
\text { radioactivity } \\
\text { applied }\end{array}$ & $\begin{array}{c}\text { Fluctuations } \\
\text { of tempera- } \\
\text { ture } \\
{ }^{\circ} \mathrm{C}\end{array}$ & $\begin{array}{l}\text { Weather } \\
\text { conditions }\end{array}$ \\
\hline
\end{tabular}

Experiment 1963

\begin{tabular}{r|l|l|l|l|l|l}
\hline I & 47 & 0,088 & 1,0 & 63 & $26-32$ & $\mid$ \\
II & 60 & 0,065 & 1,0 & 58 & $24-30$ & sunny* \\
III & 72 & 0,06 & 1,0 & 69 & $30-35$ & \\
IV & 82 & 0,037 & 0,75 & 37 & $30-36$ & \\
\hline
\end{tabular}

Experiment 1964

\begin{tabular}{r|l|l|l|l|l|l}
\hline I & 43 & 0,09 & 1,0 & 73 & $24-31$ & \\
II & 50 & 0,09 & 1,0 & 65 & $24-30$ & \\
III & 58 & 0,09 & 1,0 & 69 & $26-32$ & sunny* \\
IV & 68 & 0,09 & 1,0 & 65 & $30-33$ & \\
V & 79 & 0,07 & 0,75 & 55 & $26-33$ & \\
\hline
\end{tabular}

* About $30-35$ thousand luxes inside the box

The radioactive plant organs, killed at $-60^{\circ} \mathrm{C}$ were homogenized. Total radioactivity as well as the radioactivity of the $80 \%$ ethanol (and water) - soluble fraction were determined by means of the infinitely thin layer technique. The data obtained at each exposure were referred to the specific activity of ${ }^{14} \mathrm{CO}_{2}$ applied at the first exposure in the 1963 as the 1964 experiments.

The number of replicates was: for dry matter determination -4 pots, for chlorophyll and radioactivity determination $2 \times 2$ and $3 \times 2$ plants, respectively. 


\section{RESULTS}

The two varieties investigated in both experiments differed from each other as regards dry weight increments and the rate of tillering (Tables 2 and 3). Var. Popularna showed a higher weight of leaf laminae and sheaths as well as the ear. but particularly wide differences, in comparison with var. Chłopicka, are manifest in the weight of the culm*, mainly of its three upper internodes (Figs. 1 and 2);

Table 2

Weight of wheat $-\mathrm{g}$ d.m. per plant

Experiment 1963

\begin{tabular}{|c|c|c|c|c|c|c|c|c|c|}
\hline \multirow{3}{*}{ 窇 } & \multirow{3}{*}{$\begin{array}{c}\text { Age } \\
\text { of } \\
\text { plants } \\
\text { days }\end{array}$} & \multicolumn{4}{|c|}{ Low nitrogen dose } & \multicolumn{4}{|c|}{ High nitrogen dose } \\
\hline & & \multicolumn{2}{|c|}{ main shoot ${ }^{-}$} & \multicolumn{2}{|c|}{ tillers ${ }^{1}$ ) } & \multicolumn{2}{|c|}{ main shoot } & \multicolumn{2}{|c|}{ tillers ${ }^{1}$ ) } \\
\hline & & total & $\begin{array}{c}\text { grain }^{2} \text { ) } \\
\text { only }\end{array}$ & total & $\begin{array}{c}\text { grain }^{3} \text { ) } \\
\text { only }\end{array}$ & total & $\begin{array}{c}\text { grain }^{2} \text { ) } \\
\text { only }\end{array}$ & total & $\begin{array}{c}\text { grain }^{3} \text { ) } \\
\text { only }\end{array}$ \\
\hline \multirow{6}{*}{ 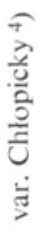 } & 43 & 1.62 & & 0.57 & & 1.55 & & 0.75 & \\
\hline & 54 & 2.70 & & 0.62 & & 2.50 & & 1.24 & \\
\hline & 67 & 3.76 & 0.38 & 0.91 & 0.04 & 3.69 & 0.41 & 2.10 & 0.09 \\
\hline & 77 & 4.47 & 1.20 & 1.09 & 0.14 & 4.34 & 1.30 & 2.63 & 0.47 \\
\hline & 86 & 4.35 & 1.66 & 1.37 & 0.39 & 4.79 & 2.00 & 2.90 & 0.89 \\
\hline & 93 & 4.27 & 1.71 & 1.35 & 0.42 & 4.80 & 2.05 & 3.10 & 1.15 \\
\hline \multirow{6}{*}{ 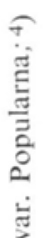 } & 43 & 1.93 & & 0.52 & & 1.70 & & 0.66 & \\
\hline & 54 & 3.19 & & 0.51 & & 3.07 & & 1.06 & \\
\hline & 67 & 4.41 & 0.37 & 0.44 & & 4.53 & 0.40 & 1.64 & 0.03 \\
\hline & 77 & 5.50 & 1.28 & 0.36 & & 5.63 & 1.59 & 2.37 & 0.30 \\
\hline & 86 & 5.39 & 1.97 & 0.58 & 0.07 & 5.77 & 2.30 & 2.86 & 0.80 \\
\hline & 93 & $5.40^{*}$ & $2.08^{*}$ & $0.60^{*}$ & $0.08 *$ & $5.70 *$ & $2.34^{*}$ & 3.19 & $0.88 *$ \\
\hline
\end{tabular}

1) Number of fertile tillers in Chłopicka: low $\mathrm{N}-0.6$; high $\mathrm{N}$ dose $-1,3$; in Popularna 0.1 and 1.1 respectively. The weight of sterile tillers varied between -0.30 (first sampling) and $0.13 \mathrm{~g}$. (last sampling);

2) Number of grains in Chłopicka, low $\mathrm{N}-41$; high $\mathrm{N}$ dose -48 ; in Popularna 45 and 50 respectively;

$\left.{ }^{3}\right)$ Number of grains in Chłopicka; low $\mathrm{N}-12$; high $\mathrm{N}$ dose -31 ; in Popularna 1 and 25, respectively.

4) Total $\mathrm{N}$ content in the main shoot in Chłopicka: low $\mathrm{N}$ dose -19 (first sampling) and $34 \mathrm{mg}$ (last sampling); high $\mathrm{N}$ dose -44 and $49 \mathrm{mg}$ respectively; in Popularna: low $\mathrm{N}$ dose -29 and $42 \mathrm{mg}$, high $\mathrm{N}$ dose -50 and $63 \mathrm{mg}$.

* Differences between varieties statistically significant $(p=0,05)$.

In both varieties the temporary weight increase of the culm after heading was due first of all to changes in the second and third internodes. The rate of tillering in Chłopicka was much higher than in Popularna, especially at low N level (in 1963).

It is worth stressing that the significantly higher production of fertile tillers in 1964 (due mainly to the smaller number of plants per pot and higher $\mathrm{N}$ supply per plant) was in Popularna accompanied by a much lower growth rate of the main

* In experiment 1963 the length of the main shoot culm at both $\mathrm{N}$ levels was the same in both varieties, accounting for $117-118 \mathrm{~cm}$; in 1964 its length $(113 \mathrm{~cm})$ in, Popularna' exceeded somewhat that in ,Chłopicka' $(109 \mathrm{~cm})$. 


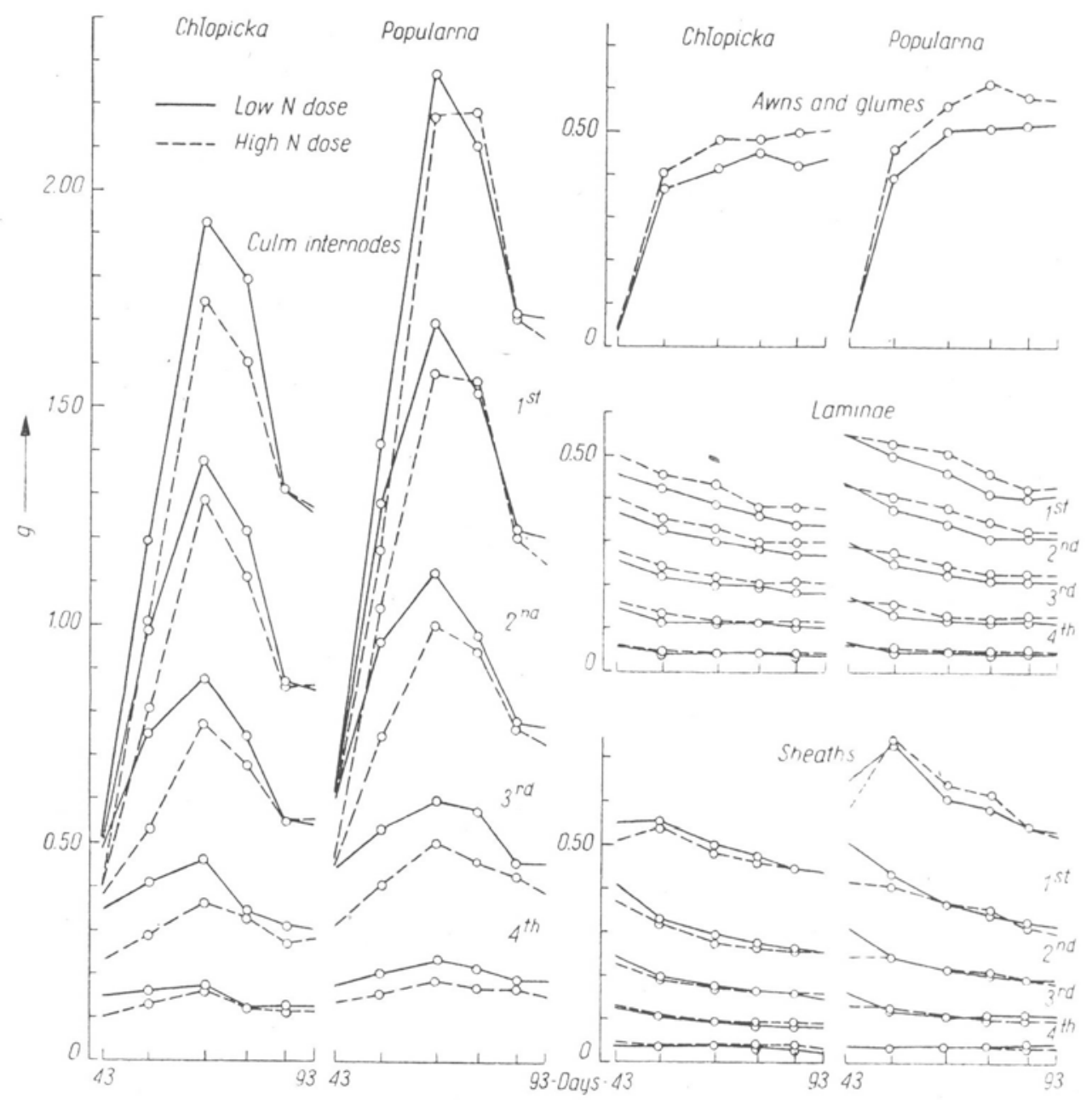

Fig. 1. Dry weight of the main shoot. Experiment 1963

* The lowest lines represent the four lowest internodes, laminae or sheaths. First $=$ the upper-most. 1 st -4 th: the distances between corresponding lines indicate the weights of particular internodes, laminae or sheaths.

shoot as compared to that in 1963, whereas the growth of the main shoot of Chłopicka was only slightly affected.

The rate of ageing of the vegetative organs of the two varieties was different in spite of the fact that ripening occurred at a similar time; this was manifested in changes in chlorophyll content, especially in 1963 (Table 4, Fig. 3). All green parts (except the ear) - especially the two uppermost leaves and internodes - of Popularna contained at each sampling more chlorophyll than those of Chłopicka. The greater green lamina area in Popularna was due mainly to differences in senescence of the lower leaves (Table 5). Some differences between varieties in chlorophyll content per $\mathrm{dm}^{2}$ with age were also found (Table 9). 


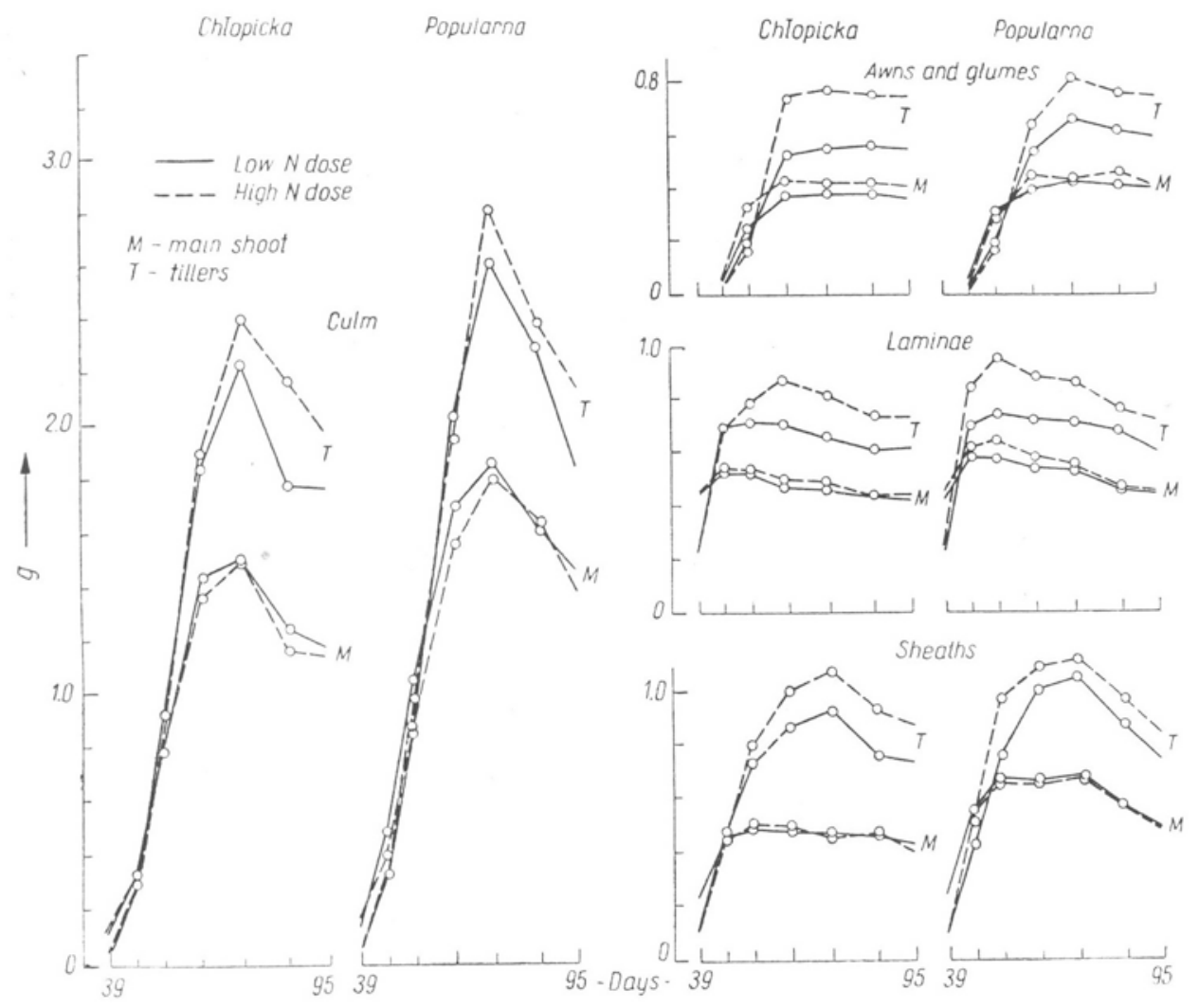

Fig. 2. Dry weight of the plant. Experiment 1964.

In 1964 the chlorophyll content in the leaves of Chłopicka was much higher (Table 6); this was due not only to a higher leaf area duration, but also to a greater content per area unit (Table 10). In the first period after heading, Chłopicka did not differ from Popularna in the amount of chlorophyll (at low $\mathrm{N}$ level). However, in the later period differences in favor of the latter were also significani.

It should be noted that, whereas in 1963 the differences between the investigated varieties in total dry weight increment occurred not only before but also after heading, in 1964 they were significant only before ear emergence. In 1963 the grain yield in Popularna was higher than in Chłopicka, in 1964 the differences in this respect between varieties were statistically insignificant (in the main shoot and the first tiller).

The influence of a higher $\mathrm{N}$ dose applied at shooting in 1963 was revealed in an increase in chlorophyll content in all green plant parts, leaf area duration, in the weight of leaf laminae and ears. The final total weight of the culm as well as of the sheaths of the main shoot was not affected; however, the increment of lower internodes was smaller, perhaps due to more intensive tillering. The influence of $\mathrm{N}$ on Chłopicka was much greater than on Popularna. 
Table 3

Weight of wheat $-\mathrm{g}$ d. m. per plant

Experiment 1964

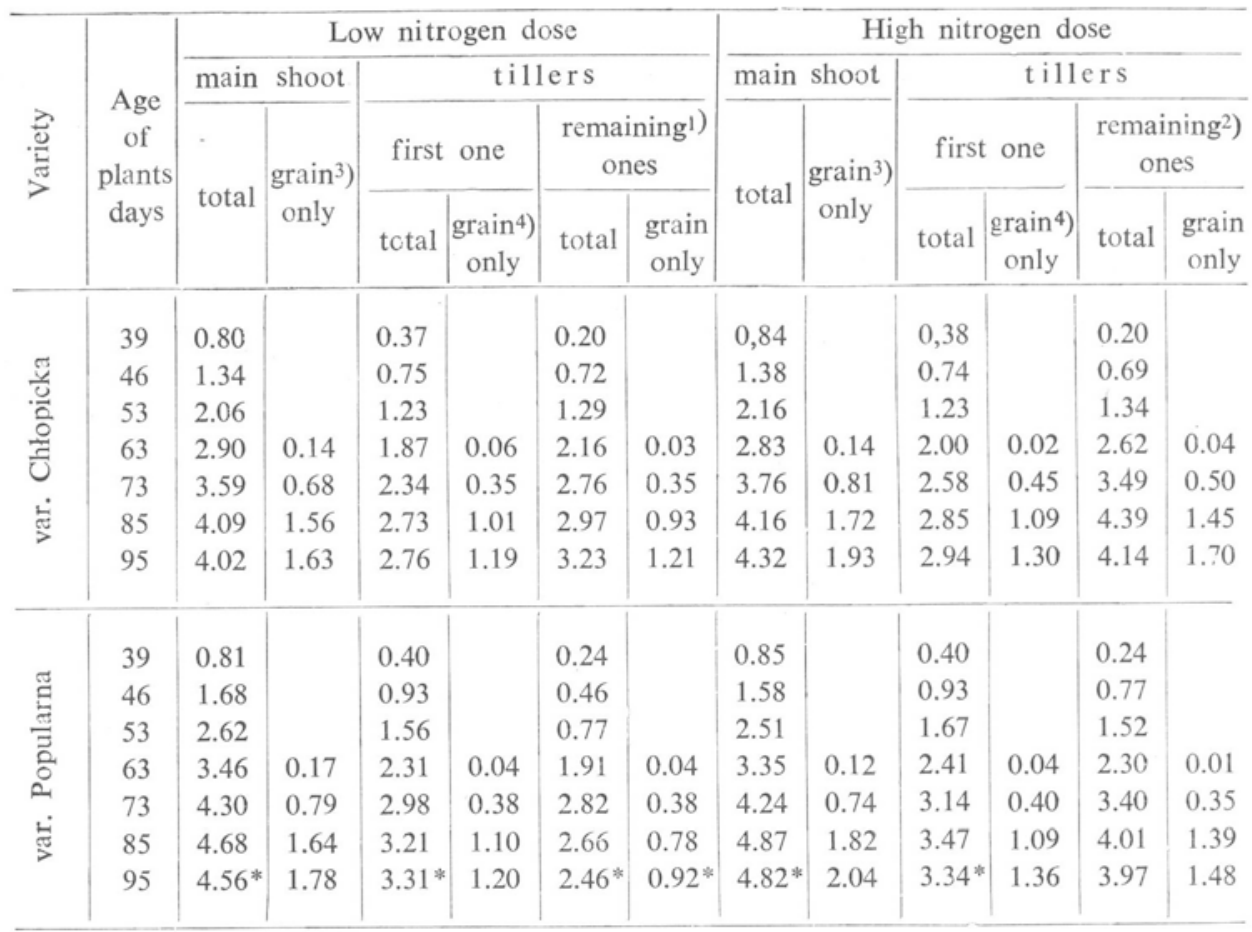

1) Number of fertile tillers in var. Chłopicka 1.63; in var. Popularna 1.26

2) ,, ,, ,, ,, ,, ,, ,, $1.89 ;,, \quad, \quad,, \quad 1.60$

3) Number of grains on both nitrogen doses in var. Chlopicka 39; in var. Popularna 38.

$\left.{ }^{4}\right)$ Number of grains in var. Chlopicka: low N-31, high N-35; in var. Popularna on both $\mathrm{N}$ doses-28.

* Differences between varieties statistically significant $(\mathrm{p}=0.05)$.

In both varieties the grain yield at high $\mathrm{N}$ level was significantly higher.

In 1964 the influence of $\mathrm{N}$ on Chłopicka was much weaker, especially as regards chlorophyll content. However, like in 1963, at later stages of development, the higher $\mathrm{N}$ dose delayed significantly senescence not only of the vegetative organs but also of the ear of the main shoot. In both varieties the weight of the grain of this shoot was higher as compared to that at low $\mathrm{N}$ level. However, the main influence of the high $\mathrm{N}$ dose was revealed in the weight of the remaining tillers (but not the first one).

Plants with their flag leaf or uppermost internode shaded - introduced additionally into the box at exposures II and III in 1963 - did not show any significant differences as compared to the unshaded ones in the radioactivity of the ear and only traces of ${ }^{14} \mathrm{C}$ in the covered part of their culm were found. However - as indicated by the data obtained a small amount of ${ }^{14} \mathrm{C}$ was translocated during the first exposure (before ear emergence) to the ear and culm (Fig. 4). 

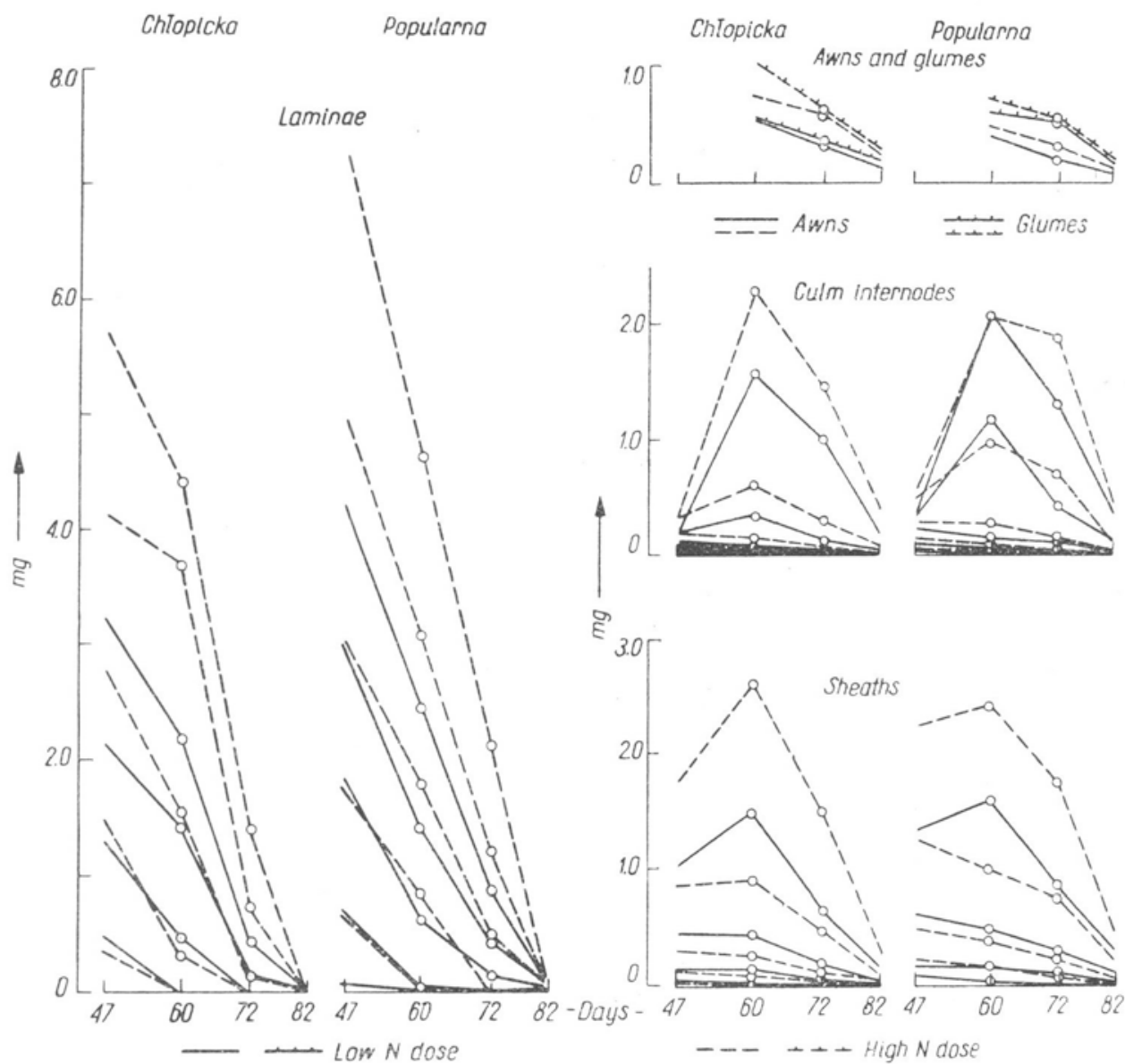

Fig. 3. Chlorophyll content in green organs of the main shoot. Experiment 1963 For notations see fig. 4.

The total plant photosynthetic activity was greatly influenced by the higher $\mathrm{N}$ supply. This was manifest especially in Chłopicka (main shoot) at later stages after heading (Table 7). The higher photosynthetic activity was due to a high extent to the greater leaf area; at the last stage the contribution of the culm as well as of the ear also increased under the influence of N. In 1964 it was Popularna, which responded stronger to the higher $\mathrm{N}$ dose than Chłopicka (Table 8, Fig. 5); this is reflected especially in the radioactivity of the first tiller (Fig. 6). The strong influence of $\mathrm{N}$ on the remaining tillers is mainly connected with their number greater than at low $\mathrm{N}$ level.

The influence of $\mathrm{N}$ on the photosynthesis rate in leaves as related to senescence, chlorophyll content and varietal differences is illustrated by the data presented in table 9. Under the experimental conditions, the maximum rate of photosynthesis about $13 \mathrm{mg} \mathrm{CO}_{2} \mathrm{dm}^{-2} . \mathrm{h}^{-1}$-was reached at a $3.2 \mathrm{mg} . \mathrm{dm}^{-2}$ chlorophyll content 
Table 4

Chlorophyll content $-\mathrm{mg}$ per plant.

Experiment 1963

\begin{tabular}{|c|c|c|c|c|c|c|c|c|c|}
\hline \multirow{3}{*}{$\underset{\text { dose }}{\mathrm{N}}$} & \multirow{3}{*}{ Plant parts } & \multicolumn{4}{|c|}{ var. Chłopicka } & \multicolumn{4}{|c|}{ var. Popularna } \\
\hline & & \multicolumn{8}{|c|}{ Age of plants - days } \\
\hline & & 47 & 60 & 72 & 82 & 47 & 60 & 72 & 82 \\
\hline \multirow{8}{*}{ Low } & main shoot & & & & & & & & \\
\hline & laminae & 3,2 & 2,2 & 0,4 & - & 4,2 & 2.5 & 0.9 & trac. \\
\hline & sheaths & 1,0 & 1,5 & 0.7 & 0.2 & 1.3 & 1.6 & 0.9 & 0.3 \\
\hline & culm & 0,2 & 0.6 & 1.1 & 0.2 & 0.3 & 2.1 & 1.3 & 0.4 \\
\hline & ear & 0.1 & 1.2 & 0.9 & 0.4 & 0.2 & 1.1 & 0.8 & 0.3 \\
\hline & grain & & & 0.2 & 0.2 & & & 0.3 & 0.3 \\
\hline & total & 4.5 & 6.5 & 3.3 & 1.0 & 6.0 & 7.3 & 4.2 & 1.3 \\
\hline & tillers & 2.5 & 0.8 & 0.9 & 0.4 & 1.4 & 0.9 & 0.2 & 0.1 \\
\hline \multirow{8}{*}{ High } & main shoot & & & & & & & & \\
\hline & laminae & 5.7 & 4.4 & 1.4 & - & 7.3 & 4.6 & 2.1 & 0.1 \\
\hline & sheaths & 1.7 & 2.6 & 1.5 & 0.3 & 2.2 & 2.4 & 1.8 & 0.5 \\
\hline & culm & 0.3 & 2.3 & 1.5 & 0.4 & 0.5 & 2.1 & 2.0 & 0.5 \\
\hline & ear & 0.3 & 1.7 & 1.3 & 0.6 & 0.2 & 1.3 & 1.0 & 0.4 \\
\hline & grain & & & 0.3 & 0.3 & & & 0.4 & 0.4 \\
\hline & total & 8.0 & 11.0 & 6.0 & 1.6 & 10.2 & 10.4 & 7.3 & 1.9 \\
\hline & tillers & 4.3 & 5.4 & 4.5 & 1.6 & 5.9 & 5.3 & 3.2 & 1.4 \\
\hline
\end{tabular}

Table 5

Lamina area of the main shoot

Experiment 1963

\begin{tabular}{|c|c|c|c|c|c|c|c|c|c|c|}
\hline \multirow{4}{*}{ Variety } & \multirow{4}{*}{$\begin{array}{c}\mathrm{N} \\
\text { dose }\end{array}$} & \multicolumn{9}{|c|}{ Age of plants - days } \\
\hline & & \multicolumn{3}{|c|}{47} & \multicolumn{3}{|c|}{60} & \multicolumn{3}{|c|}{72} \\
\hline & & \multirow{2}{*}{$\begin{array}{c}\text { number } \\
\text { of } \\
\text { leaves }\end{array}$} & \multicolumn{2}{|c|}{ area $-\mathrm{cm}^{2}$} & \multirow{2}{*}{$\begin{array}{c}\text { number } \\
\text { of } \\
\text { leaves }\end{array}$} & \multicolumn{2}{|c|}{ area $-\mathrm{cm}^{2}$} & \multirow{2}{*}{$\begin{array}{c}\text { number } \\
\text { of } \\
\text { leaves }\end{array}$} & \multicolumn{2}{|c|}{ area $-\mathrm{cm}^{2}$} \\
\hline & & & total & green & & total & green & & total & green \\
\hline \multirow{2}{*}{ Chłopicka } & low & 4 & 128 & 126 & 3 & 94 & 86 & 2 & 59 & 34 \\
\hline & high & 5 & 157 & 154 & 4 & 128 & 112 & 3 & 96 & 71 \\
\hline \multirow{2}{*}{$\begin{array}{c}\text { Popular- } \\
\text { na* }\end{array}$} & low & 5 & 153 & 139 & 4 & 130 & 103 & 3 & 95 & 63 \\
\hline & high & 5 & 168 & 180 & 5 & 165 & 151 & 3 & 97 & 87 \\
\hline
\end{tabular}

* In 82 days old plants green leaf area amounted to $4 \mathrm{~cm}^{2}$ in both treatments. 
Table 6

Chlorophyll content $-\mathrm{mg}$ per plant

Experiment 1964

\begin{tabular}{|c|c|c|c|c|c|c|c|c|c|c|c|c|c|}
\hline \multirow{5}{*}{$\begin{array}{c}\mathrm{N} \\
\text { dose }\end{array}$} & \multirow{5}{*}{$\begin{array}{l}\text { Plant } \\
\text { parts }\end{array}$} & \multicolumn{6}{|c|}{ var. Chłopicka } & \multicolumn{6}{|c|}{ var. Popularna } \\
\hline & & \multicolumn{12}{|c|}{ age of plants-days } \\
\hline & & \multicolumn{3}{|c|}{58} & \multicolumn{3}{|c|}{79} & \multicolumn{3}{|c|}{58} & \multicolumn{3}{|c|}{79} \\
\hline & & \multirow{2}{*}{ 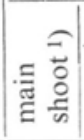 } & \multicolumn{2}{|c|}{ tillers ${ }^{3}$ ) } & \multirow{2}{*}{ 宽 } & \multicolumn{2}{|c|}{ tillers } & \multirow[b]{2}{*}{ 怘 } & \multicolumn{2}{|c|}{ tillers } & \multirow[b]{2}{*}{ 胥 } & \multicolumn{2}{|c|}{ tillers } \\
\hline & & & 荧 & غ્ઁ் & & 葾 & $\dot{\Xi ்}$ & & 芯 & 灾 & & 岕 & غ் \\
\hline \multirow{6}{*}{ Low } & laminae & 5.3 & 4.4 & 5.8 & 0.5 & 0.3 & 0.7 & 5.3 & 4.0 & 3.6 & 0.7 & 0.5 & 0.6 \\
\hline & sheaths & 1.6 & 1.6 & 1.8 & 0.6 & 0.6 & 0.8 & 1.8 & 1.6 & 1.2 & 0.7 & 0.6 & 0.8 \\
\hline & culm & 1.0 & 0.4 & 0.5 & 1.0 & 0.8 & 1.1 & 1.1 & 0.8 & 0.4 & 1.3 & 1.1 & 1.2 \\
\hline & ear & 1.1 & 0.5 & 0.4 & 0.7 & 0.6 & 0.8 & 0.9 & 0.7 & 0.3 & 0.6 & 0.6 & 0.5 \\
\hline & grain & & & & 0.1 & 0.1 & 0.2 & & & & 0.2 & 0.2 & 0.2 \\
\hline & total & 9.0 & 6.9 & 8.5 & 2.9 & 2.4 & 3.6 & 9.1 & 7.1 & 5.5 & 3.5 & 3.0 & 3.3 \\
\hline \multirow{6}{*}{ High } & laminae & 6.5 & 4.6 & 7.4 & 1.0 & 1.3 & 1.7 & 9.1 & 5.9 & 8.0 & 2.4 & 1.9 & 2.7 \\
\hline & sheaths & 2.5 & 2.2 & 3.0 & 1.6 & 1.5 & 2.2 & 2.9 & 2.2 & 2.7 & 1.8 & 1.3 & 1.8 \\
\hline & culm & 1.7 & 0.7 & 0.8 & 1.6 & 1.4 & 2.2 & 1.4 & 0.7 & 0.4 & 2.0 & 1.7 & 2.5 \\
\hline & ear & 1.4 & 0.6 & 0.8 & 1.1 & 1.1 & 1.7 & 1.0 & 0.6 & 0.4 & 0.7 & 0.7 & 1.0 \\
\hline & grain & & & & 0.2 & 0.2 & 0.3 & & & & 0.2 & 0.3 & 0.2 \\
\hline & total & 12.1 & 8.1 & 12.0 & 5.5 & 5.5 & 8.1 & 14.4 & 9.4 & 11.5 & 7.1 & 5.9 & 8.2 \\
\hline
\end{tabular}

1) Four green leaves in both varieties and both treatments

2) Two green leaves in both varieties and both treatments; on high $\mathrm{N}$ dose the third one contains only traces of chlorophyll

${ }^{3}$ ) rem. - remaining tillers

Table 7

Plant radioactivity immediately after exposure to ${ }^{14} \mathrm{CO}_{2}$ and at ripeness $-10^{3} \mathrm{cpm}$ per plant Experiment 1963

\begin{tabular}{|c|c|c|c|c|c|c|c|c|c|c|c|c|c|c|}
\hline \multirow[b]{4}{*}{$\begin{array}{l}\frac{3}{2} \\
\frac{0}{\pi} \\
>\end{array}$} & \multirow{4}{*}{$\begin{array}{l}0 \\
0 \\
0 \\
z \\
z\end{array}$} & \multirow{4}{*}{ 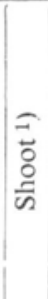 } & \multicolumn{12}{|c|}{${ }^{14} \mathrm{CO}_{2}$ assimilated on the } \\
\hline & & & \multicolumn{3}{|c|}{$47^{\text {th }}$ day $-I$} & \multicolumn{3}{|c|}{$60^{\text {th }}$ day - II } & \multicolumn{3}{|c|}{$72^{\text {nd }}$ day - III } & \multicolumn{3}{|c|}{$82^{\text {nd }}$ day - IV } \\
\hline & & & \multirow[b]{2}{*}{$\begin{array}{l}\text { imme- } \\
\text { dia- } \\
\text { tely }\end{array}$} & \multicolumn{2}{|c|}{ at ripeness } & \multirow[b]{2}{*}{$\begin{array}{c}\text { imme } \\
\text { dia- } \\
\text { tely }\end{array}$} & \multicolumn{2}{|c|}{ at ripeness } & \multirow[b]{2}{*}{$\begin{array}{l}\text { imme- } \\
\text { dia- } \\
\text { tely }\end{array}$} & \multicolumn{2}{|c|}{ at ripeness } & \multirow[b]{2}{*}{$\begin{array}{c}\text { imme- } \\
\text { dia- } \\
\text { tely } 2 \text { ) }\end{array}$} & \multicolumn{2}{|c|}{ at ripeness } \\
\hline & & & & total & $\begin{array}{l}\text { in } \\
\text { grain } \\
\% \text { of } \\
\text { total }\end{array}$ & & total & $\begin{array}{l}\text { in } \\
\text { grain } \\
\% \text { of } \\
\text { total }\end{array}$ & & total & $\begin{array}{l}\text { in } \\
\text { grain } \\
\% \text { of } \\
\text { total }\end{array}$ & & total & $\begin{array}{l}\text { in } \\
\text { grain } \\
\% \text { of } \\
\text { total }\end{array}$ \\
\hline \multirow{4}{*}{$\begin{array}{l}\frac{\pi}{0} \\
\frac{\partial}{0} \\
\frac{\pi}{0}\end{array}$} & \multirow{2}{*}{ low } & M & 510 & 321 & 13 & 412 & 238 & 56 & 300 & 167 & 89 & 69 & 48 & 86 \\
\hline & & $\mathrm{T}$ & 169 & 100 & 12 & 106 & 51 & 25 & 110 & 41 & 76 & 57 & 18 & 90 \\
\hline & \multirow{2}{*}{ high } & M & 599 & 380 & 10 & 498 & 278 & 56 & 434 & 276 & 89 & 167 & 128 & 87 \\
\hline & & $\mathrm{T}$ & 327 & 147 & 8 & 239 & 131 & 14 & 257 & 135 & 77 & 236 & 125 & 88 \\
\hline \multirow{4}{*}{ 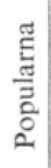 } & \multirow{2}{*}{ low } & M & 543 & 333 & 10 & 483 & 307 & 38 & 530 & 299 & 86 & 160 & 130 & 90 \\
\hline & & $\mathrm{T}$ & 175 & 92 & 11 & 94 & 20 & 26 & - & - & - & - & - & - \\
\hline & \multirow{2}{*}{ high } & M & 678 & 387 & 8 & 584 & 339 & 40 & 619 & 320 & 86 & 226 & 146 & 88 \\
\hline & & $\mathrm{T}$ & 319 & 136 & 7 & 230 & 119 & 10 & 259 & 110 & -70 & 209 & 87 & 89 \\
\hline
\end{tabular}

1) $\mathrm{M}$ - main shoot; $\mathrm{T}$ - tillers

$\left.{ }^{2}\right)$ Radioactivity of main shoot leaves in Chlopicka: low $\mathrm{N}$ dose -10 , high $\mathrm{N}$ dose $-56 \times 10^{3} \mathrm{cpm}$, in Popularna -48 and $72 \times 10^{3} \mathrm{cpm}$, respectively. 


\section{Chlopicka}
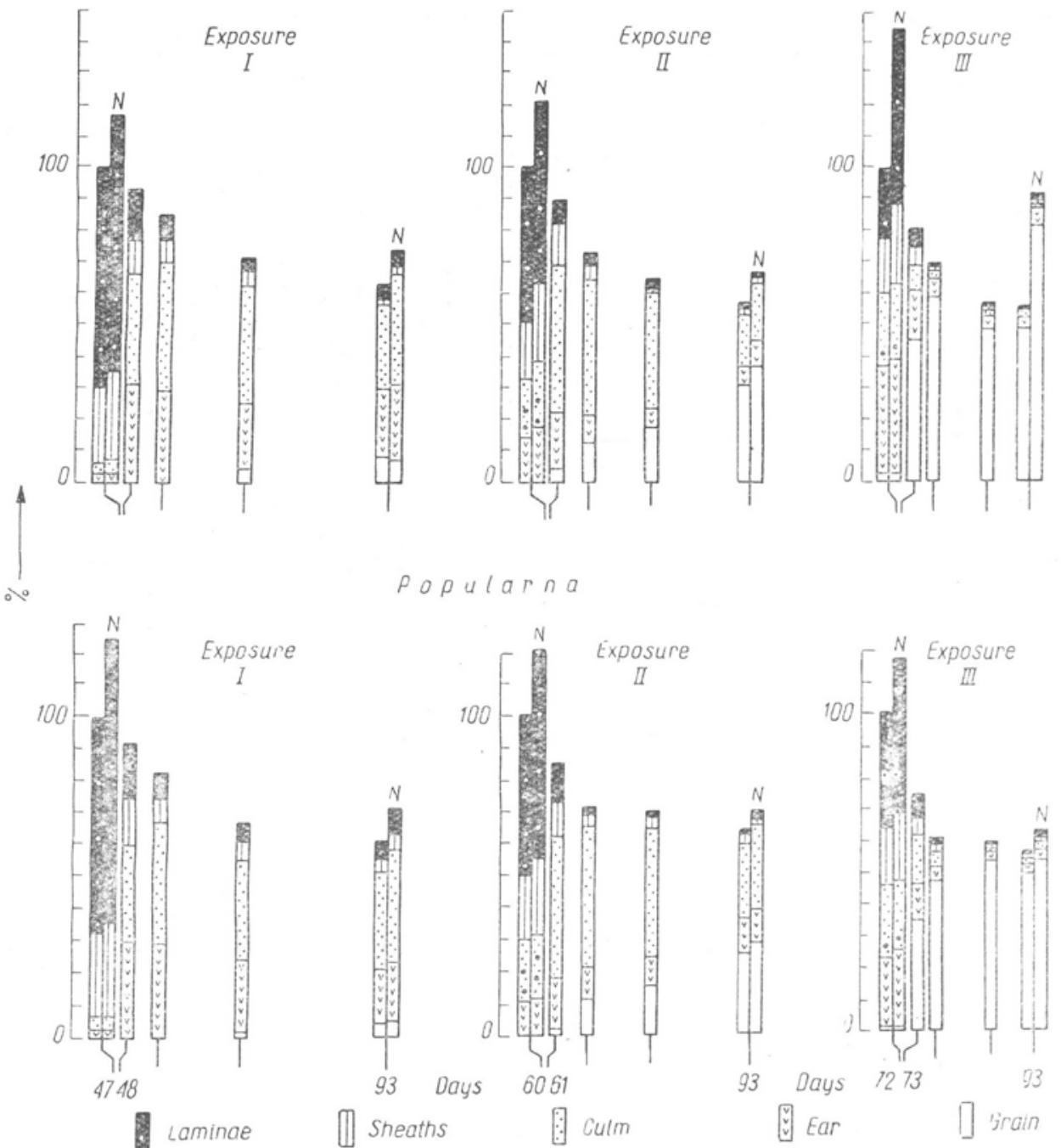

Fig. 4. Changes in radioactivity of the main shoot after exposure to ${ }^{14} \mathrm{CO}_{2}$ (total radioactivity of the main shoot at low $\mathrm{N}$ level immediately after exposure - 100\%). Experiment 1963.

In this experiment as well as in expt. 1964 (first and last sampling) the bars denoted $\mathrm{N}$ represent piants at high $\mathrm{N}$ level. Radioactivity of the successive laminae and internodes immediately after exposure are indicated by white and black points, respectively.

(2-nd leaf of 47-days-old plants of Chłopicka in 1963). An increase in chlorophyll even in the flag leaf was not associated with an increase in the photosynthesis rate. Unfortunately some differences in $\mathrm{CO}_{2}$ concentration, temperature and especially in time of exposure $(20-30 \mathrm{~min})$, do not allow to compare plants of different ages. However, it is seen that $\mathrm{N}$ caused a higher conten of chlorophyll per unit area in 


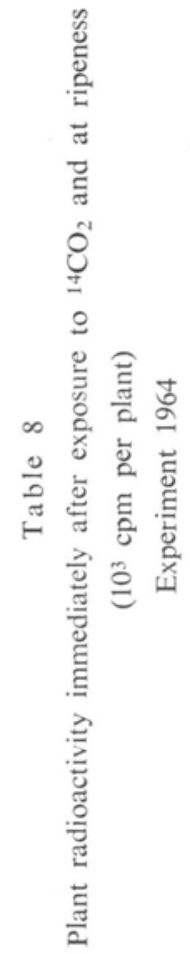

\begin{tabular}{|c|c|c|c|c|c|c|c|c|c|c|}
\hline \multirow{3}{*}{ 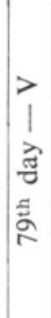 } & \multirow{2}{*}{ 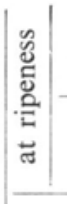 } & \multirow{2}{*}{ 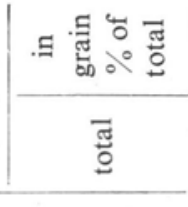 } & \multirow{2}{*}{ 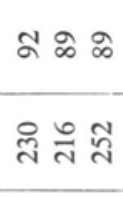 } & \multirow{2}{*}{$\mid$} & \multirow{2}{*}{ 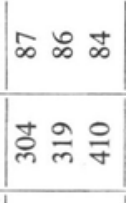 } & \multirow{2}{*}{\multicolumn{2}{|c|}{ 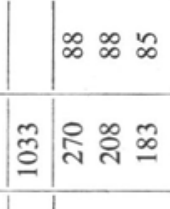 }} & \multirow{2}{*}{\multicolumn{2}{|c|}{ 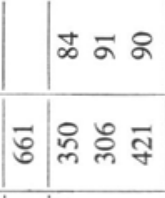 }} & \multirow{2}{*}{$\frac{1}{10}$} \\
\hline & & & & & & & & & & \\
\hline & & 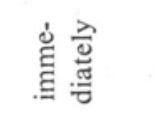 & 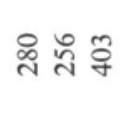 & बे & ल) & $\underset{\sim}{\approx}$ & ल્ల స̃ సิ & $\tilde{\tilde{\lambda}}$ & 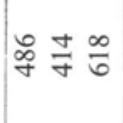 & $\stackrel{\infty}{n}$ \\
\hline \multirow{3}{*}{ 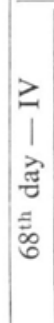 } & \multirow{3}{*}{ 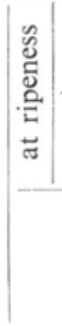 } & 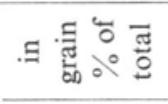 & $\bar{\infty} \stackrel{\infty}{\infty}$ & & $\infty$ iి & & か & & $\cong 8 \%$ & \\
\hline & & ฐँ & ले సे & đু & 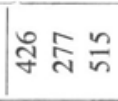 & $\frac{\infty}{\simeq}$ & సี సี స̊ సి & $\mathbb{N}$ & ઠ્ర స్ సి సે & $\stackrel{\infty}{\text { ป }}$ \\
\hline & & 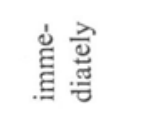 & ஜั రి & ఏ̊ & 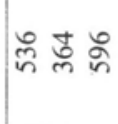 & 竎 & ஓ શે થે & ลั. & 守 & ڤ్ \\
\hline \multirow{3}{*}{ 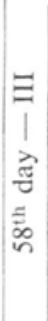 } & \multirow{3}{*}{ 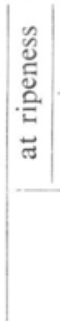 } & 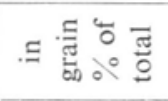 & 워으 & & $m \equiv I$ & & 워 $\mathrm{m}$ & & $m \cong a$ & \\
\hline & & ฐే & 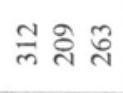 & ‡্ & लె స్ స్ సे & $\hat{\kappa}$ & $\stackrel{\infty}{m} \frac{0}{\sim} \stackrel{\infty}{n}$ & $\tilde{A}$ & 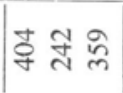 & 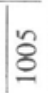 \\
\hline & & 冚 & \& $\bar{్}$ & $\hat{\varrho}$ & 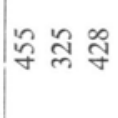 & 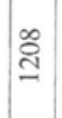 & 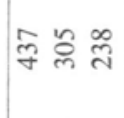 & ஓ & 壳勇 & $\sqrt[n]{2}$ \\
\hline \multirow{3}{*}{ 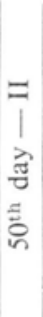 } & \multirow{3}{*}{ 氞 } & $\Xi$ 焉。ํㅀ & $=\infty r$ & & $a \infty 0$ & & 으 & & $\infty \sim 0$ & \\
\hline & & ฐ̃ & 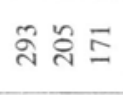 & ठे & ๙ิণ స్ సิ & $\hat{\curvearrowright}$ & 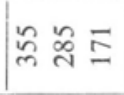 & $\bar{\infty}$ & ભૂ సి స్ & 于 \\
\hline & & 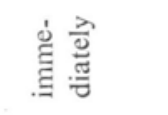 & స్ల్ల స్ స్ & $\underset{\sim}{\infty}$ & ঋ্ণ & สู & : & ळ & 瓦导字 & $\bar{\sim}$ \\
\hline \multirow{3}{*}{ 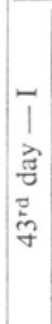 } & \multirow{2}{*}{ 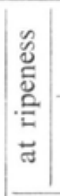 } & . & ron & & rr & & $r N n$ & & $\infty a \infty$ & \\
\hline & & ङ્ّ & ঠ్ల $\cong$ & ț & $\bar{ల} \precsim \bar{్}$ & I & 商吉 & $\grave{N}$ & త্ల స్ స్ల & గ్ర \\
\hline & & 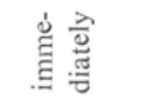 & 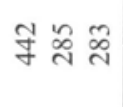 & 응 & ণ ర్ల ల్లా & $\stackrel{\infty}{\infty}$ & : ర్రి & I & 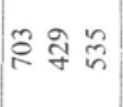 & $\underline{0}$ \\
\hline \multicolumn{3}{|c|}{ (I sloous } & 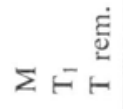 & 吾 & $\sum F^{-}$ & ב: & 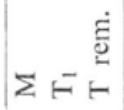 & ฐٓ & $\sum \forall \stackrel{\dot{\Xi}}{\Sigma}$ & ङ \\
\hline \multicolumn{3}{|c|}{ әsop N } & \multicolumn{2}{|l|}{ MOI } & \multicolumn{2}{|l|}{ ૫ริ!̣! } & \multicolumn{2}{|l|}{ MOI } & \multicolumn{2}{|l|}{ ૫ฮิ!บ } \\
\hline \multicolumn{3}{|c|}{ Кџə!ฺ中 } & \multicolumn{4}{|c|}{ Ехㄲ악 } & \multicolumn{4}{|c|}{ euse[ndo } \\
\hline
\end{tabular}




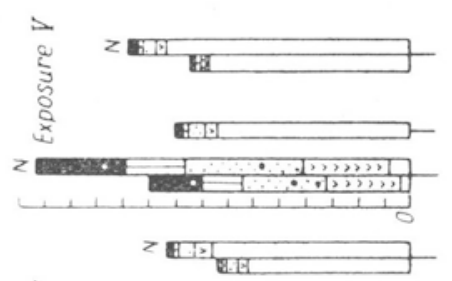

$\gtrless$
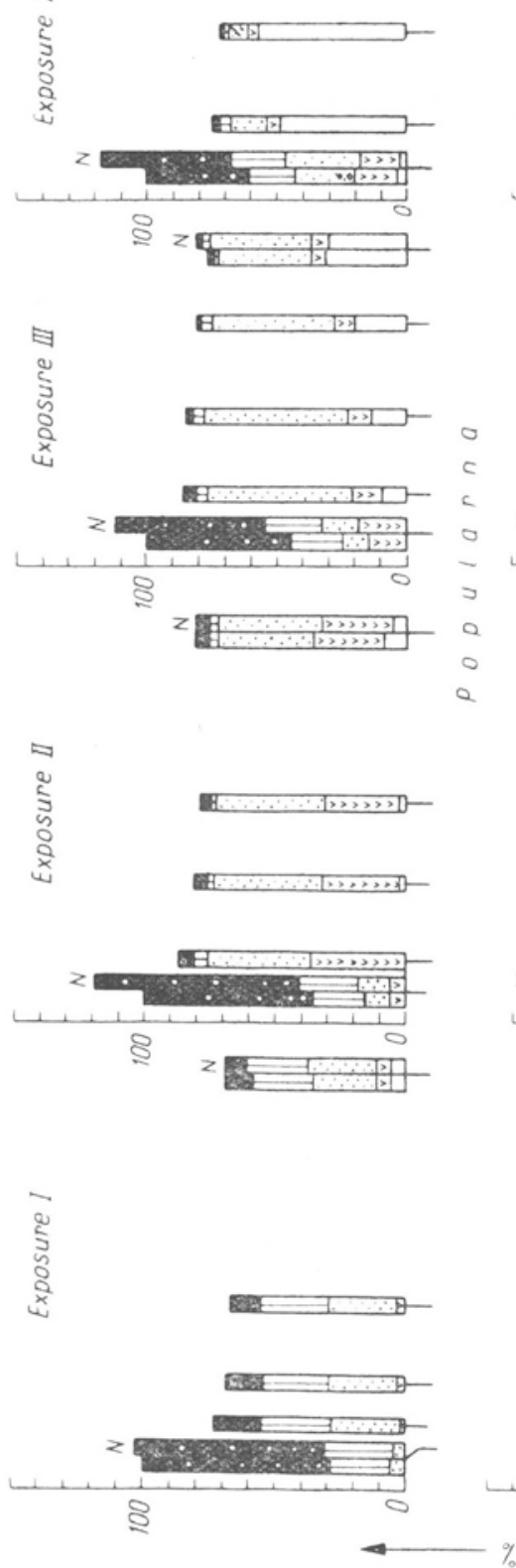
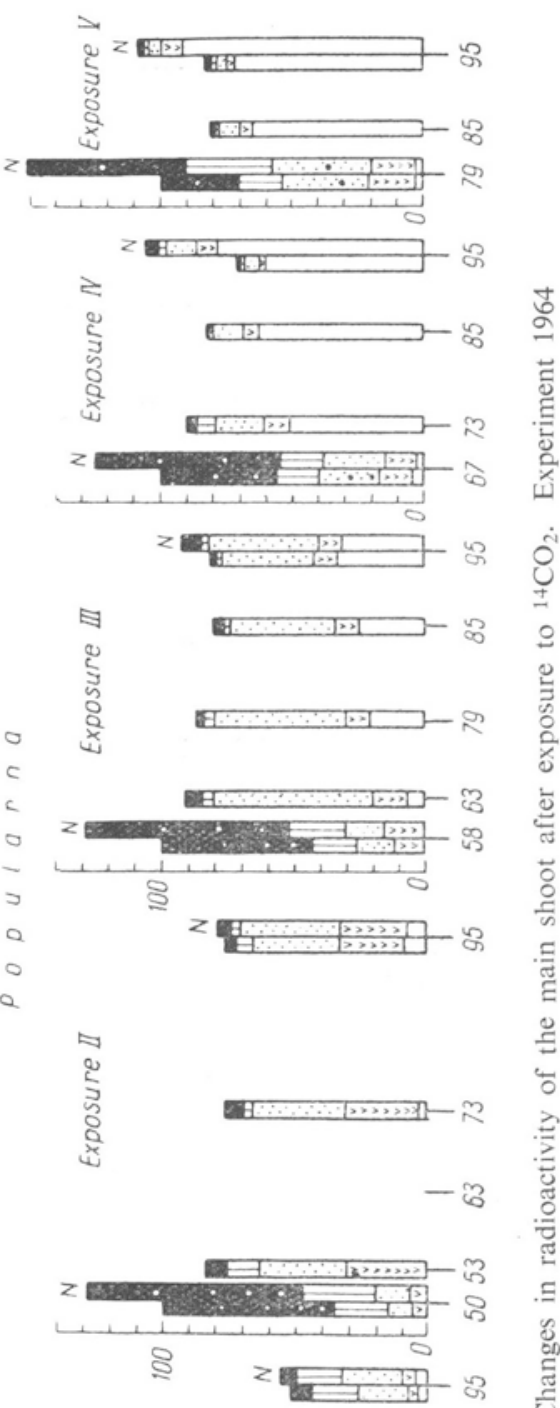

in

in

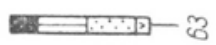

as $7 \ldots 10$

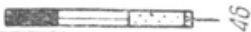

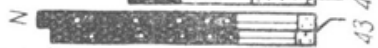

ธ 

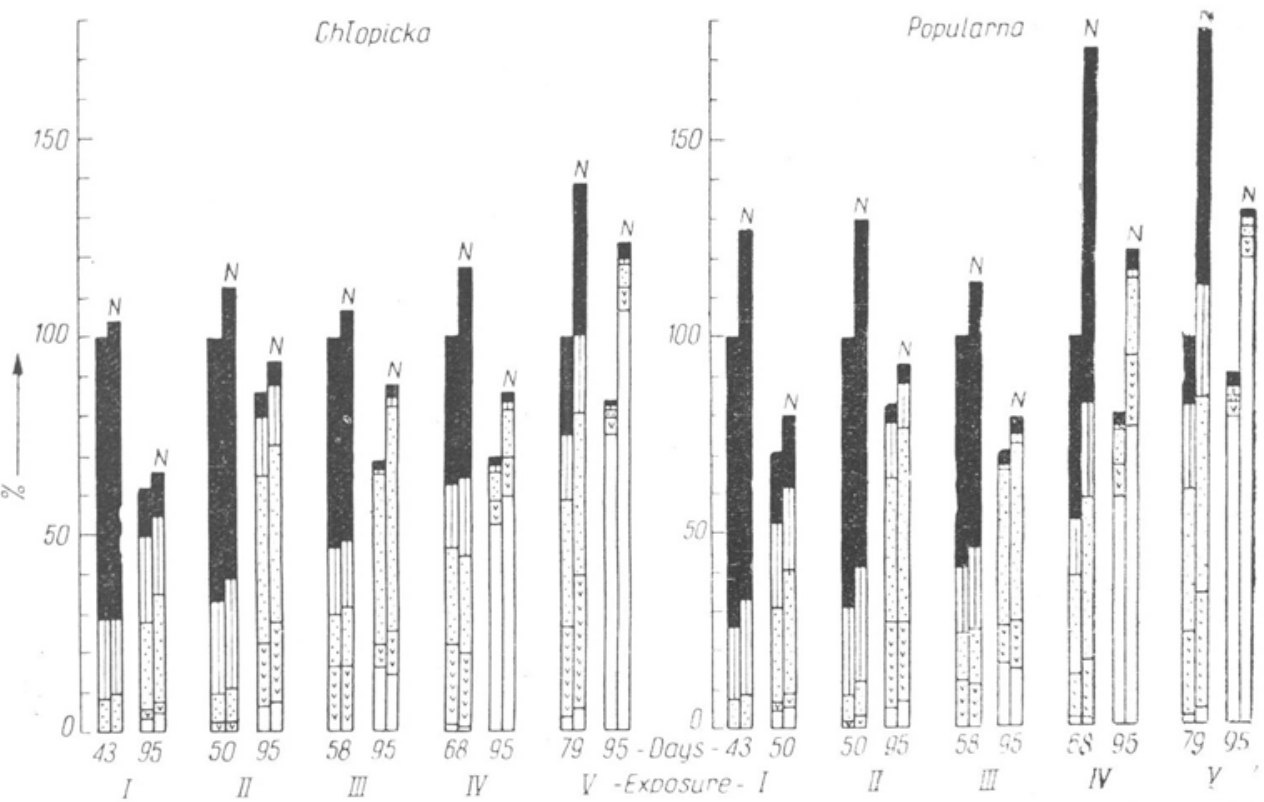

Fig. 6. Radioactivity of the first tiller immediately after exposure and at ripeness. Experiment 1964 For notations see fig. 4 .

all leaves during the whole time under investigation; but its influence on the rate of photosynthesis was not always associated with its influence on chlorophyll content.

At each exposure the rate of photosynthesis was lower in older leaves than in younger ones.

Table 9

Chlorophyll content (mg) and ${ }^{14} \mathrm{C}$ assimilated $\left(10^{3} \mathrm{cpm}\right)$ per $\mathrm{dm}^{2}$ of green lamina area (main shoot) Experiment 1963

\begin{tabular}{|c|c|c|c|c|c|c|c|c|c|c|c|c|c|}
\hline \multirow{4}{*}{$\stackrel{\mathrm{N}}{\text { dose }}$} & \multirow{4}{*}{$\begin{array}{c}\text { Lami- } \\
\text { na* }\end{array}$} & \multicolumn{12}{|c|}{ Age of plants - days } \\
\hline & & \multicolumn{4}{|c|}{$47 * *$} & \multicolumn{4}{|c|}{60} & \multicolumn{4}{|c|}{72} \\
\hline & & \multicolumn{2}{|c|}{ Chłopicka } & \multicolumn{2}{|c|}{ Popularna } & \multicolumn{2}{|c|}{ Chłopicka } & \multicolumn{2}{|c|}{ Popularna } & \multicolumn{2}{|c|}{ Chłopicka } & \multicolumn{2}{|c|}{ Popularna } \\
\hline & & chlor. & ${ }^{14} \mathrm{C}$ & chlor. & ${ }^{14} \mathrm{C}$ & chlor. & ${ }^{14} \mathrm{C}$ & chlor. & ${ }^{14} \mathrm{C}$ & chlor. & ${ }^{14} \mathrm{C}$ & chlor. & ${ }^{14} \mathrm{C}$ \\
\hline \multirow{4}{*}{ low. } & $1^{\text {st }}$ & 4.0 & 295 & 4.5 & 305 & 3.0 & 230 & 3.2 & 270 & 1.5 & 243 & 1.9 & 328 \\
\hline & $2^{\text {nd }}$ & 3.2 & 283 & 3.5 & 282 & 2.7 & 206 & 2.6 & 241 & 1.1 & 77 & 1.2 & 301 \\
\hline & $3^{\text {rd }}$ & 2.3 & 220 & 3.4 & 268 & 1.3 & 147 & 1.5 & 140 & & & 0.9 & 140 \\
\hline & $4^{\text {th }}$ & 1.4 & 151 & 2.0 & 184 & & & & & & & & \\
\hline \multirow{4}{*}{ high } & $1^{\text {st }}$ & 6.0 & 311 & 6.9 & 315 & 5.1 & 275 & 4.8 & 265 & 3.0 & 296 & 3.3 & 306 \\
\hline & $2^{\text {nd }}$ & 4.3 & 294 & 5.1 & 284 & 3.6 & 266 & 3.7 & 253 & 1.9 & 202 & 2.3 & 292 \\
\hline & $3^{\mathrm{rd}}$ & 3.5 & 258 & 3.2 & 263 & 3.2 & 160 & 2.5 & 191 & 0.8 & 135 & 1.8 & 161 \\
\hline & $4^{\text {th }}$ & 3.2 & 160 & 2.7 & 189 & & & & & & & & \\
\hline
\end{tabular}

* 1st - lamina of the flag leaf

** $300.10^{3} \mathrm{cpm}$ corresponds to about $14 \mathrm{mg} \mathrm{CO}_{2} \mathrm{dm}^{-2} . \mathrm{h}^{-1}$ 
Table 10

Chlorophyll content $(\mathrm{mg})$ and ${ }^{14} \mathrm{C}$ assimilated $\left(10^{3} \mathrm{cpm}\right)$ per $\mathrm{dm}^{2}$ of green lamina area (main shoot)

Experiment 1964

\begin{tabular}{|c|c|c|c|c|c|c|c|c|c|}
\hline \multirow{4}{*}{$\stackrel{\mathrm{N}}{\text { dose }}$} & \multirow{4}{*}{ Lamina* } & \multicolumn{8}{|c|}{ Age of plants - days } \\
\hline & & \multicolumn{4}{|c|}{58} & \multicolumn{4}{|c|}{79} \\
\hline & & \multicolumn{2}{|c|}{ Chłopicka } & \multicolumn{2}{|c|}{ Popularna } & \multicolumn{2}{|c|}{ Chłopicka } & \multicolumn{2}{|c|}{ Popularna } \\
\hline & & chlor. & ${ }^{14} \mathrm{C}$ & chlor. & ${ }^{14} \mathrm{C}$ & chlor & ${ }^{14} \mathrm{C}$ & chlor. & ${ }^{14} \mathrm{C}$ \\
\hline \multirow{2}{*}{ Low. } & $1^{\text {st }}$ & 5.8 & $280 * *$ & 5.1 & 302 & 0.9 & 137 & 0.8 & 165 \\
\hline & $2^{\text {nd }}$ & 5.5 & 209 & 5.6 & 251 & & & & \\
\hline \multirow{2}{*}{ High } & $1^{\text {st }}$ & 6.6 & 274 & 7.8 & 305 & 2.0 & 210 & 2.9 & 269 \\
\hline & $2^{\text {nd }}$ & 6.4 & 206 & 8.0 & 237 & & & & \\
\hline
\end{tabular}

* 1st - lamina of the flag leaf

** This value corresponds to about $15 \mathrm{mg} \mathrm{CO}_{2} \mathrm{dm}^{-2} \cdot \mathrm{h}^{-1}$

Table 11

Contribution of particular green parts to total photosynthetic activity of the main shoot $(\%)$.

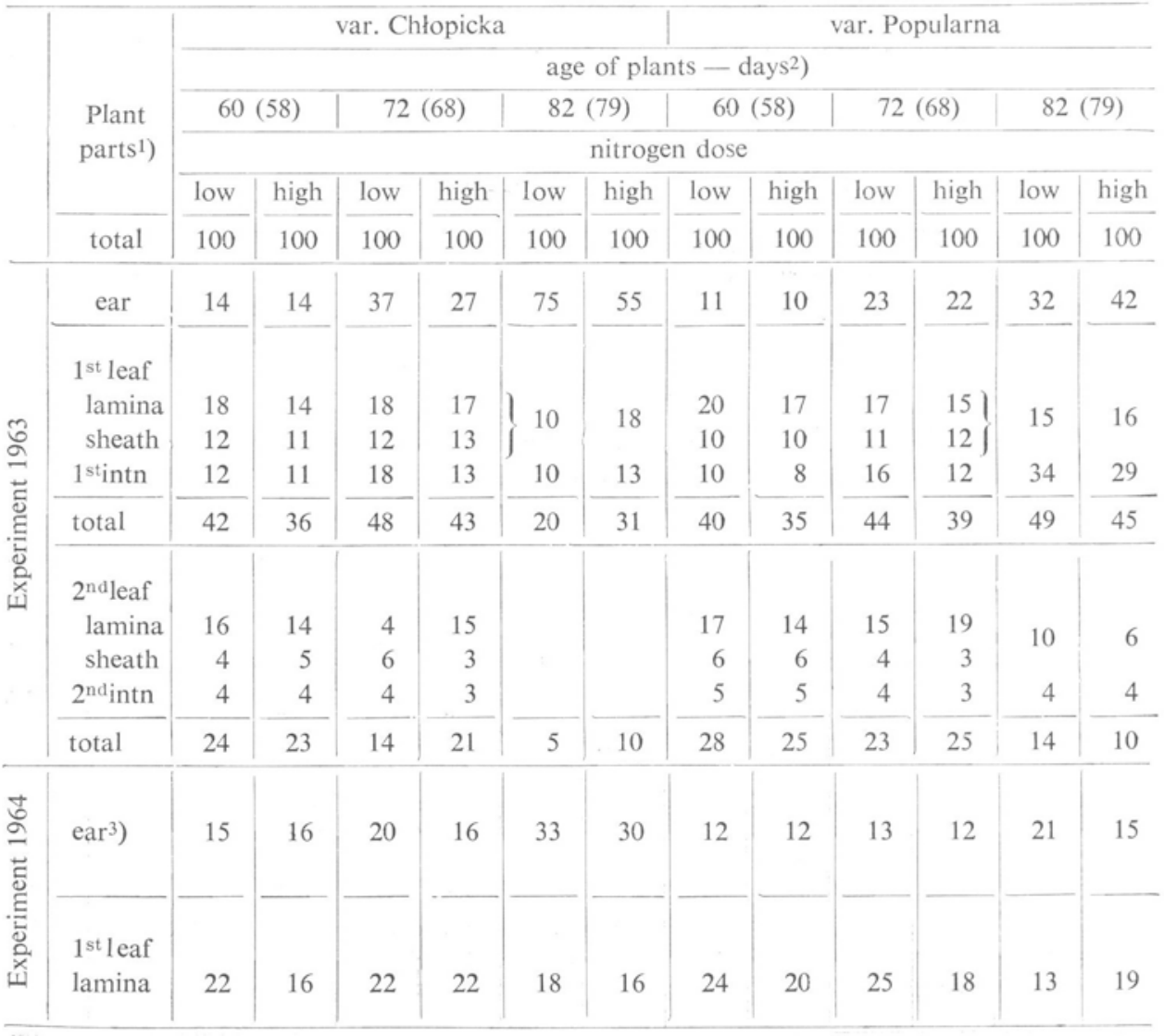

1) intn. - internode; 1 st - uppermost

${ }^{2}$ ) in parentheses - age of plants in expt. 1964

${ }^{3}$ ) the contribution of the ear of the first tiller to its total photosynthetic activity was similar to that found in the main shoot. 
The difference depending on the leaf age (insertion order) was much more pronounced in Chłopicka than in Popularna. At low $\mathrm{N}$ dose the rate of photosynthesis in the leaves of Chłopicka was lower than in analogous leaves of Popularna - two upper leaf laminae of the youngest plants excepted - even when no or insignificant differences in chlorophyll content (below $4 \mathrm{mg} . \mathrm{dm}^{-2}$ ) occurred. In Popularna the higher $\mathrm{N}$ dose had in most cases no significant effect on the rate of photosynthesis, even when it caused marked differences in chlorophyll content (within the range below $3 \mathrm{mg} . \mathrm{dm}^{-2}$ ). In Chłopicka the high $\mathrm{N}$ dose caused an increase in the rate of photosynthesis; its influence was more pronounced in relatively not very old leaves. Under the influence of $\mathrm{N}$ the leaves of Chłopicka reached very often the same level of photosynthesis rate as those of Popularna, but never exceeded it.

The data obtained in 1964 (Table 10) show that differences in the rate of photosynthesis between the investigated varieties may occur even when the content of chlorophyll is relatively very high. It is worth noting that, at late stages of development, also Popularna (flag leaf) responded in its photosynthesis rate to $\mathrm{N}$ applied at shooting. It does not seem that the differences in photosynthesis rate between leaves of various insertion order - except perhaps in exposure I in 1963 - were mainly due to differences in their illumination (the light intensity within the area of the 2-3 upper leaves was rather uniform in the box). It should be added that neither differences in the chlor. a/chlor. b ratio could be suspected to be responsible for the differences between the varieties in photosynthesis rate with ageing. Acccording to so far unpublished data there are no significant differences between them in this respect; in plants of both varieties immediately after heading the chlor. a/chlor $\mathrm{b}$ ratio in the flag-leaf lamina was $2,1-2,2$; in the third one $1,9-2,0$; ten days later $2,0-2,1$ and 1,8 respectively.

The coniribution of the particular green parts to the total photosynthetic activity of the shoots changed greatly depending on the plant age, $\mathrm{N}$ supply and variety.

Before ear emergence in both varieties the contribution of leaf laminae accounted for about $70 \%$ at both $\mathrm{N}$ levels, the contribution of the two uppermost leaves constituting only about one half of this value. At anthesis the share of leaf laminae was still high accounting in 1963 for about $50 \%$, in 1964 for $55-60 \%$ in the main shoot as well as in the first tiller and being a little higher in Popularna. In the later period their share diminished rapidly, especially in Chłopicka in 1963.

The data presented in table 11 show that, at anthesis, the contribution of the ear to the total photosynthetic activity of the shoot was rather small amounting in Chłopicka to $14-16$, in Popularna $10-12 \%$. Its contribution in the following period depended on the rate of senescence, different in both varieties especially in 1963. In the last period, the contribution of the ear in Chłopicka was 75 , whereas in Popularna only $32 \%$. Differences - but not so wide - in this respect were manifest also in 1964 experiment, in which the highest contribution in Chłopicka amounted to $33 \%$. The relatively high efficiency of ear chlorophyll in photosynthesis is worth stressing (table 12). At high $\mathrm{N}$ level - in contrast to that of the flag leaf lamina - it did not decrease; this is perhaps due to the unchanged relative content of chlorophyll (per g dry matter) of the glumes and awns. 
It should be added that in spite of the higher weight of the green parts of the ear in Popularna, especially in 1963 their photosynthetic activity was lower or equal to that found in the ear of Chłopicka; when calculated per unit of chlorophyll content there were no significant differences between the varieties in this respect.

Similarly as in previous investigations on wheat, translocation of assimilates from leaf laminae and sheaths (unless they were used in the grow.h process in situ exposure I 1964) was rapid before and after ear emergence.

Table 12

${ }^{14} \mathrm{CO}_{2}$ assimilated by the ear and the flag leaf lamina $-10^{3} \mathrm{cpm}$ per $\mathrm{mg}$ of chlorophyll

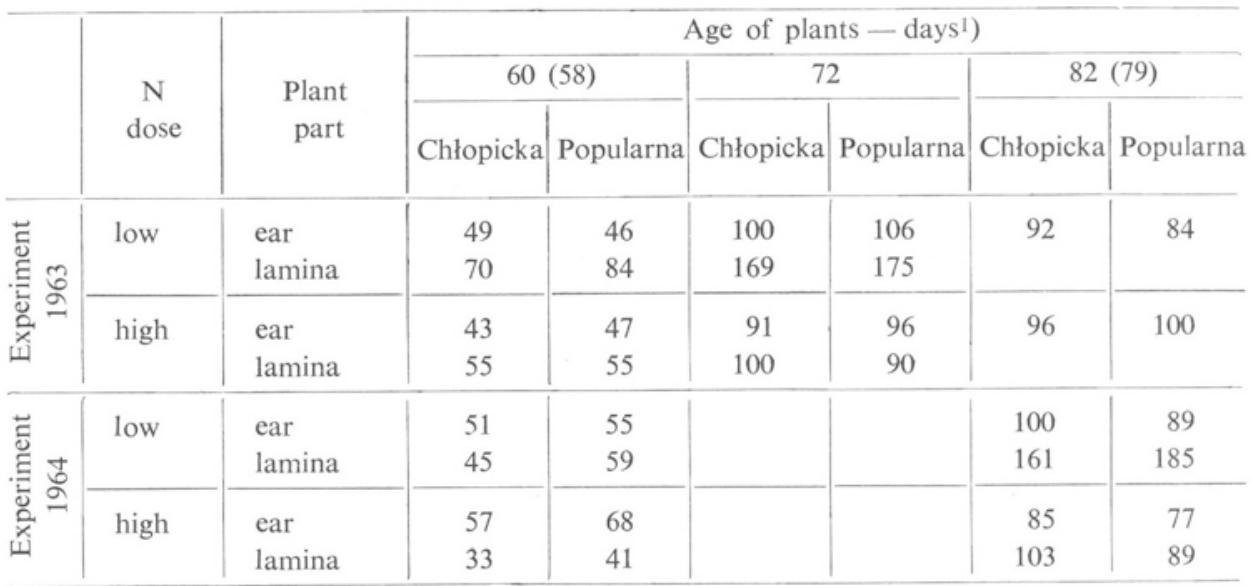

1) In parentheses - age of plants in expt. 1964

The culm plays a double role - as an acceptor of assimilates utilizing them in growth, and simultanously as a temporary store for them. A great proportion of carbon assimilated not only before heading but also between ear emergence and anthesis were incorporated into this organ. The contribution of assimilates formed at the end of this period constituted in 1963 in Chłopicka at low $\mathrm{N}$ about 27, at high $\mathrm{N}$ level $30 \%$ of the total ${ }^{14} \mathrm{C}$ found at ripening (at low $\mathrm{N}$ level); in Popularna it constituted 37 and $41 \%$ respectively. In 1964 it was relatively higher, in Chłopicka 47 and $51 \%$; in Popularna* 45 and 52\% respectively. The data presented in table $13^{* *}$ clearly show that especially the second and third internodes are capable of accumulating in a soluble form a relatively large amount of assimilates formed before and immediately after heading. Their translocation to the grain occurs mainly at the stage of intensive grain filling. Changes in the total radioactivity and in the radioactivity of the soluble fraction in green parts indicate that the amount

* When referred to the final amount of ${ }^{14} \mathrm{C}$ found in Chłopicka at low $\mathrm{N}$ level, it accounts in $1963-48$ and $53 \%$; in $1964-50$ and $58 \%$.

** In 1964 changes in assimilate distribution after exposure in the first tiller were similar to those found in the main shoot; therefore only the radioactivities immediately after exposure and at ripeness are reported (fig. 6). 
Table 13

Changes in radioactivity (total and ethanol soluble fraction) of particular parts of the main shoot after exposure to ${ }^{14} \mathrm{CO}_{2}\left(10^{3} \mathrm{cpm}\right)$ - var. Chłopicka ${ }^{1)}$

\begin{tabular}{|c|c|c|c|c|c|c|c|c|c|c|c|c|c|}
\hline \multirow{3}{*}{\multicolumn{2}{|c|}{ Exposure }} & \multirow{3}{*}{$\begin{array}{c}\text { Time } \\
\text { after } \\
\text { exposure } \\
\text { days }\end{array}$} & \multirow{3}{*}{$\begin{array}{c}\text { Radio- } \\
\text { activity²) }\end{array}$} & \multicolumn{10}{|c|}{ Plant parts } \\
\hline & & & & \multirow[b]{2}{*}{$\begin{array}{c}\text { lami- } \\
\text { nae }\end{array}$} & \multirow[b]{2}{*}{$\begin{array}{l}\text { she. } \\
\text { aths }\end{array}$} & \multicolumn{5}{|c|}{ culm-internodes ${ }^{3}$ ) } & \multicolumn{3}{|c|}{ ear } \\
\hline & & & & & & I & II & III & rem. & total & $\begin{array}{c}\text { awns } \\
\text { glu- } \\
\text { mes }\end{array}$ & rachis & grain \\
\hline \multirow{16}{*}{ 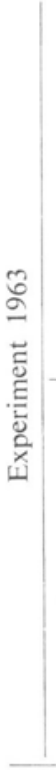 } & \multirow{8}{*}{ 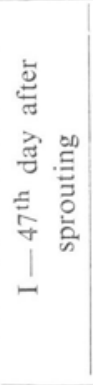 } & 1 & total & 82 & 56 & 36 & 69 & 46 & 25 & 176 & & 159 & \\
\hline & & & soluble & 45 & 29 & 10 & 22 & 18 & 14 & 64 & & 52 & \\
\hline & & 7 & total & 40 & 35 & 58 & 66 & 58 & 27 & 209 & 124 & 24 & \\
\hline & & & soluble & 14 & 14 & 5 & 12 & 24 & 14 & 55 & 18 & 3 & \\
\hline & & 20 & total & 30 & 21 & 40 & 70 & 63 & 18 & 191 & 85 & 21 & 11 \\
\hline & & & soluble & 8 & 7 & 4 & 12 & 19 & 9 & 44 & 7 & 3 & 5 \\
\hline & & 46 & total & 23 & 11 & & & & & 135 & 89 & 20 & 43 \\
\hline & & (ripeness) & soluble & 3 & 2 & & & & & 15 & 3 & 1 & 5 \\
\hline & \multirow{8}{*}{$\Xi$} & 1 & total & 34 & 54 & 87 & 70 & 28 & 8 & 193 & 62 & 13 & 16 \\
\hline & & & soluble & 22 & 36 & 57 & 51 & 17 & 7 & 132 & 42 & 11 & 7 \\
\hline & & 7 & total & 17 & 21 & 85 & 63 & 20 & 7 & 175 & 30 & 10 & 49 \\
\hline & & & soluble & 8 & 14 & 28 & 46 & 16 & 6 & 96 & 10 & 5 & 12 \\
\hline & & 17 & total & 10 & 9 & 68 & 55 & 23 & 9 & 155 & 20 & 6 & 69 \\
\hline & & & soluble & 4 & 6 & 15 & 35 & 19 & 7 & 74 & 5 & 5 & 8 \\
\hline & & 33 & total & 10 & 8 & & & & & 65 & 20 & 5 & 130 \\
\hline & & (ripeness) & soluble & 4 & 3 & & & & & 8 & 5 & 1 & 13 \\
\hline \multirow{16}{*}{ 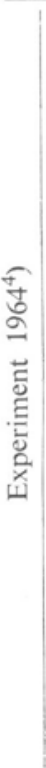 } & \multirow{8}{*}{ 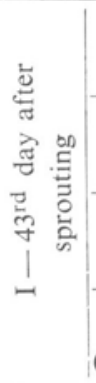 } & 3 & total & 78 & 123 & & & & & 114 & & 10 & \\
\hline & & & soluble & 41 & 41 & & & & & 26 & & & \\
\hline & & 10 & total & 60 & 104 & & & & & 114 & & 20 & \\
\hline & & & soluble & 20 & 14 & & & & & 21 & & 5 & \\
\hline & & 20 & total & 55 & 105 & 14 & & & 10 & 119 & 18 & 4 & traces \\
\hline & & & soluble & 12 & 12 & 3 & & & 4 & 20 & 2 & traces & \\
\hline & & 52 & total & 52 & 102 & & & & & 104 & 22 & 4 & 22 \\
\hline & & (ripeness) & soluble & 10 & 10 & & & & & 6 & 2 & traces & 2 \\
\hline & \multirow{8}{*}{ 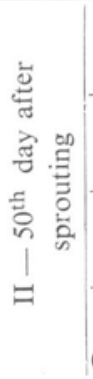 } & 3 & total & 21 & 21 & 32 & & & 20 & 142 & 100 & 32 & \\
\hline & & & soluble & 12 & 12 & 7 & & & 10 & 36 & 21 & 6 & \\
\hline & & $13^{\prime}$ & total & 18 & 12 & 29 & & & 24 & 148 & 81 & 29 & 5 \\
\hline & & & soluble & 8 & 5 & 4 & & & 6 & 24 & 7 & 2 & 1 \\
\hline & & 23 & total & 14 & 9 & 24 & & 8 & 26 & 148 & 78 & 26 & 12 \\
\hline & & & soluble & 5 & 3 & 2 & & 4 & 7 & 23 & 5 & 1 & 2 \\
\hline & & 45 & total & 15 & 9 & 26 & & 10 & & 130 & 79 & 25 & 33 \\
\hline & & (ripeness) & soluble & 4 & 3 & 1 & & & 6 & 7 & 4 & 2 & 2 \\
\hline
\end{tabular}

1) similar results were obtained with var. Popularna

${ }_{2}$ radioactivity of the water soluble fraction in laminae, sheaths and ear constituted about $87-75 \%$ of that of the ethanol soluble fractions; in culm and grain the radioactivity of both fractions was similar

3) I - the uppermost, rem. - remaining lower internodes

4) changes in ${ }^{14} \mathrm{C}$ (total and soluble) distribution in plants exposed to ${ }^{14} \mathrm{CO}_{2}$ on the 58 th day after sprouting were similar to those in plants of exposure II in 1963. 
of assimilates formed before heading and found in the grain at ripeness cannot be due in a major extent to the process of reassimilation.

After anthesis the role of the culm as well as of the ear as assimilate acceptor was much less significant. However, also at the first period of grain formation similarly as in previous investigations - some temporary accumulation of current

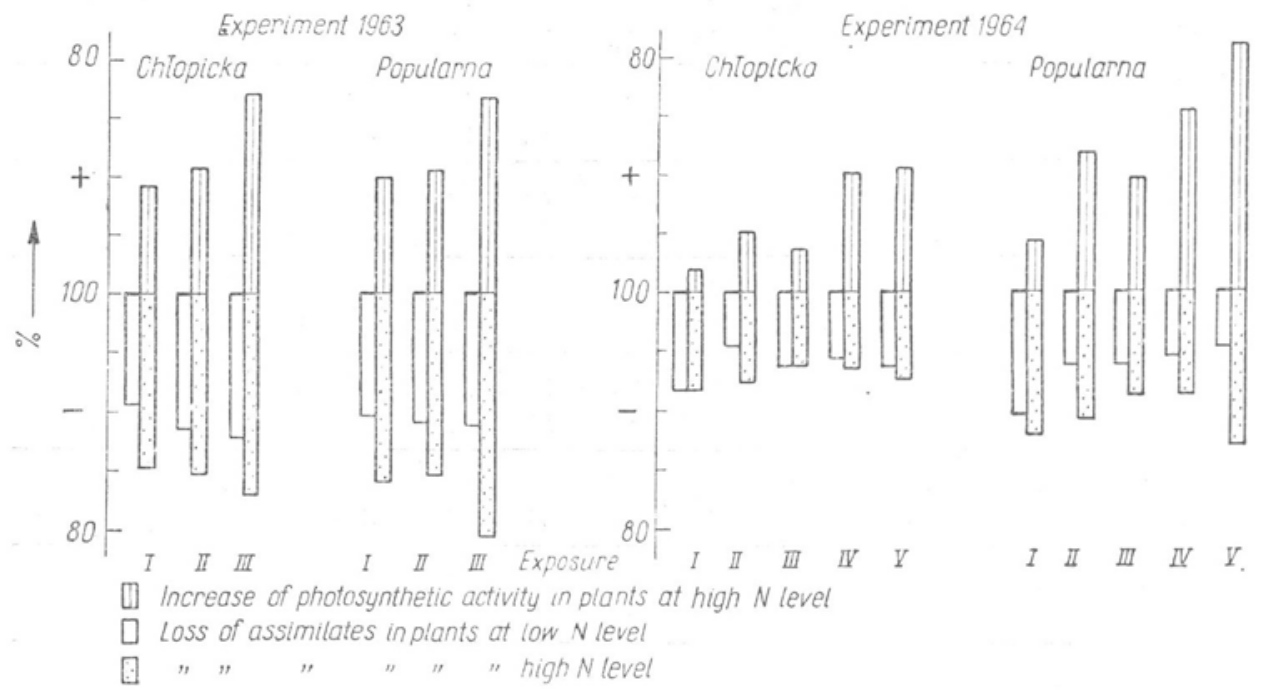

Fig. 7. Influence of nitrogen on the total plant photosynthetic activity and assimilate losses in the aerial parts (total ${ }^{7}$ radioactivity found immediately after exposure to ${ }^{14} \mathrm{CO}_{2}$ in plants at low $\mathrm{N}$ level $-100 \%$ )

photosynthesis products in the culm could be observed. Assimilate losses from the aerial plant parts were considerable not only during the first day following photosynthesis but also in the later period. In 1963 carbon losses expressed in relative values were similar in both varieties (Fig. 7); the amount of assimilates lost at high was greater than at low $\mathrm{N}$ level owing mainly to higher relative loss in tillers especially at later stages of development. However, also in the main shoot the losses were greater. Therefore the differences in the final accumulation of carbon caused by different $\mathrm{N}$ doses were small. especially when assimilates formed up to anthesis (in Popularna even later) are concerned.

In 1964 the total plant photosynthetic activity was much higher than in 1963. Therefore, perhaps, the relative losses were smaller. Also in this experiment the differences in the final accumulation of assimilates formed before anthesis depending on the $\mathrm{N}$ supply were very small in the main shoot as well as in the tillers.

The data concerning the final accumulation of assimilates in the grain are similar in both experiments. The contribution of carbon assimilated a week before ear emergence ranged between $7-12 \%$ of its total amount found at ripeness, being relatively lower in Popularna than in Chłopicka. The contribution of assimilates 
Expertment

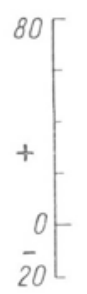

1963
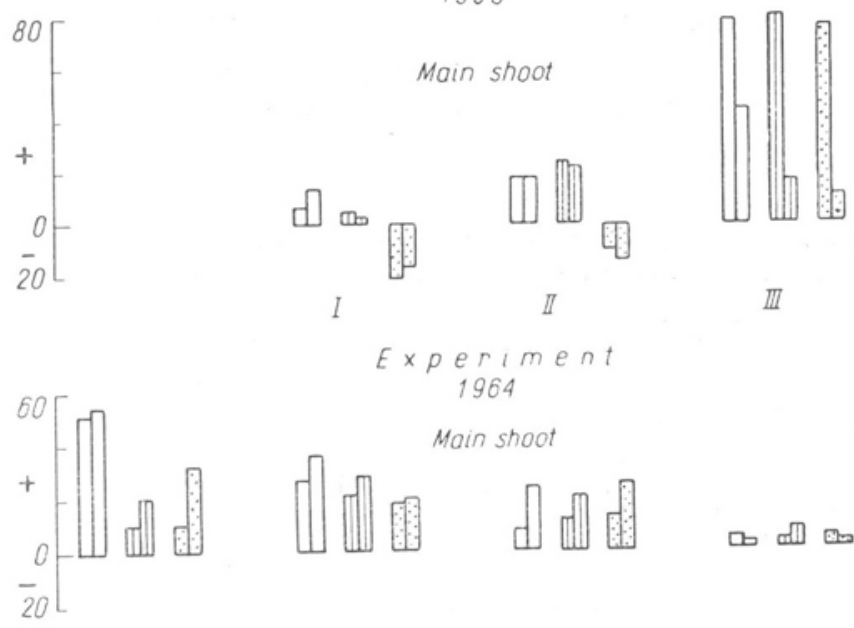

III

Exposure to ${ }^{14} \mathrm{CO}_{2}$

Main shoot
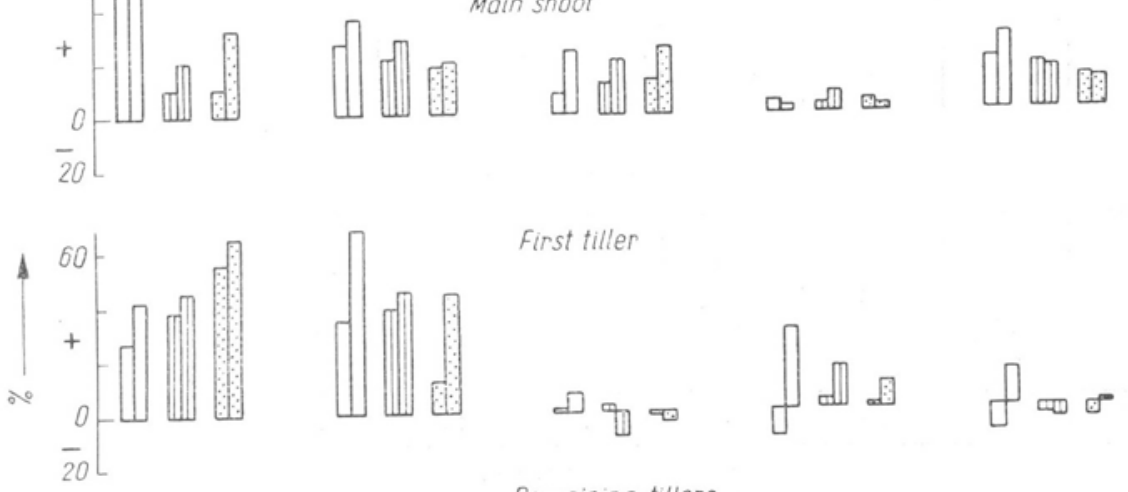

First tiller

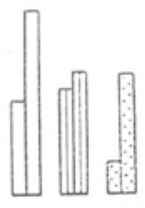

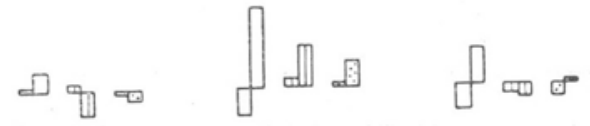<smiles>CC#CCOc1ccccc1</smiles>

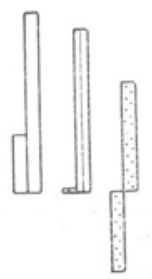

Remaining tillers
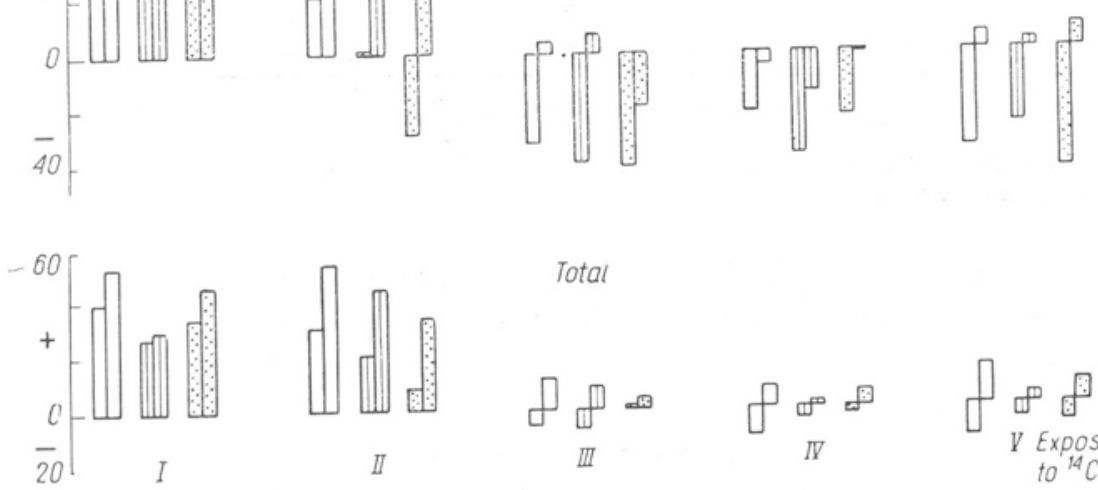

Total

$$
\begin{aligned}
& \rrbracket_{\mathbb{I I}}^{\mathbb{1 I}} \\
& \bigoplus_{I 7}^{\infty}
\end{aligned}
$$

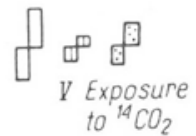

Differences $(\%)$ in total radioactivity found immediately after exposure

" " " " " at ripeness

" " " " radioactivity found in grain only at ripeness

Fig. 8. Photosynthetic activity and productivity of var. Popularna in comparison with var. Chłopicka. (first bar - low $\mathrm{N}$ dose, second bar - high $\mathrm{N}$ dose) 
formed between heading and anthesis* gradually increased up to $40-50 \%$ and of those formed during the first period of grain development to $75-80 \%$, reaching at the last stages $85-90 \%$ of the amount found at ripeness.

In both experiments differences in the $\mathrm{N}$ level had no positive influence on the final amount in grain of carbon assimilated before heading. It was mainly the culm that was the greater ,utiliser" of assimilates at the higher $\mathrm{N}$ level.

\section{Table 14}

Distribution of dry matter between organs of the main shoot at ear emegence and at ripeness (final dry weight of Chłopicka at low $\mathrm{N}$ level $-100 \%$ )

\begin{tabular}{|c|c|c|c|c|c|c|c|c|}
\hline \multirow{4}{*}{$\begin{array}{l}\text { Plant } \\
\text { parts }\end{array}$} & \multicolumn{4}{|c|}{ Experiment 1963} & \multicolumn{4}{|c|}{ Experiment 1964} \\
\hline & \multirow{2}{*}{\multicolumn{2}{|c|}{$\begin{array}{c}\text { Chłopicka } \\
\mathrm{N} \text { dose }\end{array}$}} & \multicolumn{2}{|c|}{ Popularna } & \multicolumn{2}{|c|}{ Chłopicka } & \multicolumn{2}{|c|}{ Popularna } \\
\hline & & & & lose & & & & \\
\hline & low & high & low & - high & low & high & low & high \\
\hline
\end{tabular}

at ear emergence

\begin{tabular}{l|l|l|l|l||r|r|r|r}
\hline lamina & 10 & 11 & 12 & 13 & 13 & 13 & 14 & 15 \\
sheaths & 13 & 12 & 17 & 16 & 11 & 11 & 17 & 16 \\
ear & 10 & 11 & 12 & 13 & 8 & 10 & 10 & 9 \\
culm & 28 & 24 & 33 & 30 & 20 & 19 & 25 & 22 \\
\hline total & 61 & 58 & 74 & 72 & 52 & 53 & 66 & 62 \\
\hline
\end{tabular}

at ripeness

\begin{tabular}{l|r|r|r|r||r|r|r|r}
\hline lamina & 8 & 9 & 10 & 10 & 10 & 11 & 11 & 11 \\
sheaths & 10 & 10 & 12 & 12 & 10 & 10 & 12 & 12 \\
ear $^{1}$ & 12 & 14 & 15 & 16 & 11 & 12 & 12 & 12 \\
culm & 30 & 31 & 40 & 39 & 28 & 28 & 35 & 36 \\
grain & 40 & 48 & 49 & 55 & 41 & 48 & 44 & 50 \\
\hline total & 100 & 112 & 126 & 132 & 100 & 109 & 114 & 121 \\
\hline
\end{tabular}

$\left.{ }^{1}\right)$ share of the rachis in Chłopicka accounted for about $2 \%$ in both experiments, in Popularna $3-$ in expt. 1963 and $2 \%$ in expt. 1964.

Differences caused by $\mathrm{N}$ in the final accumulation in the grain of assimilates formed between heading and anthesis were manifest only in the 1963 experiment in Chłopicka.

The data obtained clearly show that the higher grain yield at higher $\mathrm{N}$ level was due mainly to the greater final accumulation of carbon assimilated after anthesis. Thus the greater was the influence of $\mathrm{N}$ on the photosynthetic efficiency of the plants in this period, the stronger was its influence on the final assimilate accumulation in the grain; in 1963 the response of the main shoot of Chłopicka was higher in this

* Differences between the main shoot and the first tiller at exposure III in 1964 are very characteristic in this respect - table 8 , 
respect as compared with that of Popularna, in 1964 it was similar or even weaker (first tiller).

Varietal differences in photosynthetic activity and productivity depended on many factors (fig. 8). In 1963, when at low $\mathrm{N}$ level the ageing process of the main shoot of Chłopicka after anthesis was much more rapid than in Popularna (perhaps partly owing to a relatively higher rate of tillering), the preponderance of the latter in photosynthetic activity and productivity in this period was very high reaching more than $70 \%$ (exposure III) and $170 \%$ (exposure IV). At high $\mathrm{N}$ level it was incomparably lower especially as for as the final assimilate accumulation in grain is concerned. It is worth stressing that the accumulation in grain of carbon assimilated before anthesis was in Popularna in this experiment lower than in Chłopicka at both $\mathrm{N}$ levels. In the photosynthetic efficiency of tillers, Chłopicka also exceeded Popularna. In 1964, when the differences in tillering and in ageing rate were much smaller, the preponderance of Popularna was mainly manifest in the period before heading. In the later stages it exceeded significantly Chłopicka only as regards the main shoot and remained behind the latter markedly as regards the remaining tillers at low nitrogen level.

It should be noted that the final accumulation of ${ }^{14} \mathrm{C}$-assimilates, formed at all the stages investigated, in the grain was in the main shoot of Popularna somewhat higher than in that of Chłopicka*.

The differences found between varieties in photosynthesis at various stages, in assimilate loss and assimilate accumulation in various parts find partly confirmation in the distribution of dry matter between the plant organs at heading and ripeness (Table 14). The fact that in both experiments the differences between Chłopicka and Popularna in the culm weight at ripeness were higher than at heading and greater than the differences in grain yield is worth noting.

\section{DISCUSSION}

Chłopicka - the most high-yielding among spring wheat varieties in Poland - was in the last decade exceeded by a new variety Popularna in most of our agricultural regions. The latter - as seen - has a relatively low tillering capacity, high individual shoot weight and large leaf area at heading, i.e. features characteristic of the most yielding varieties investigated by de Silva (cf. Stoy 1965). On the other hand, according to v. Dobben (1962), the improvement of wheat from 1900 has been reached by increasing not the total shoot production but mainly the grain/ shoot ratio, which in spring wheat is supposed to be correlated with the number of grains per ear (Bunting et al. 1964). Differences in ear photosynthesis (Asana, Saini 1958, Lupton 1961, Buttrose 1962, Wattson et al. 1963), in leaf area duration after ear emergence or anthesis, with special regard to the flag leaf and the uppermost internode (Watson, 1956; Nichiporovich 1961; Nichiporo-

* Differences in dry weight of the grain of the main shoot as well as of the first tiller were not statistically significant. 
vich, Własowa 1961; Thorne 1962, 1965; Welbank et al. 1966) are considered as also, or mainly, responsible for varietal differences in the degree of organic matter accumulation in grain.

It seems that the physiological traits of genotypic or phenotypic character, which may be important determinants of high yielding capacity, are more complicated. Some of them are revealed in the reported experiments. First of all the data obtained show that in wheat carbon assimilated before ear emergence can-although to a relatively low extent - contribute to the organic matter accumulation in the grain. More important is the carbon assimilated between heading and anthesis. It is true, that assimilates formed after anthesis coniribute to a relatively much higher degree to grain filling; however one should take account of the fact that the plant photosynthetic activity prior to this stage is higher, so in absolute amounts, the contribution of carbon assimilated before anthesis can be of greater importance than indicated by the relative values ( 11 to $40-50 \%$ ). If so, there are two problems worth noting: 1 . the role of other leaves than the uppermost one and 2. the influence of culm growth on assimilate redistribution. Even at anthesis the photosynthetic activity of the second and the third laminae was considerable; their assimilates translocated to the lower internodes were exported partially to the grain at later stages. The extent to which they contribute to root activity is to be investigated*. It has been shown that the culm plays an important role as assimilate acceptor also between heading and anthesis. The relatively small differences in grain yield between varieties as compared to differences between them in total shoot weight are due to a significant extent to the differences in assimilate utilization for culm growth at that period. It seems worth while to pay more attention to this organ as to: 1) a photosynthates acceptor in relation to leaf photosynthetic activity and to assimilate redistribution at grain filling; 2 ) a temporary store for soluble assimilates with regard to the interdependence between its storage capacity and character of growth, 3) an assimilating organ (mainly the uppermost internode). In previous investigations on Chłopicka (Birecka, 1966) it has been found that, when culm growth is diminished by CCC, a relatively greater amount of carbon assimilated after heading is translocated partly to the ear.

It should, be added that the driving force, on which the efflux of assimilates from leaves to the culm is dependent, and by which the soluble organic compounds stored in the internodes for a relatively long time move towards the ear, is not clear. The results obtained in the reported as well as previous experiments on wheat seem to support the opinion that the efflux from the laminae is not dependent on the ,sink" capacity, partly determined by its rate of growth (Hartt et al, 1964a, b,; Wardlaw, 1965). It is hard to tell whether such a dependence, assumed by Nosberger et al. (1965), between the ear and internodes at grain filling actually exists. It seems that the redistribution of assimilates is controlled by a more complicated mechanism, which may be indirectly associated with the increasing sink capacity of the ear.

* This problem as well as that of the photosynthetic activity of lower leaves under field conditions will be discussed in more detail in another publication. 
The photosynthetic activity of the investigated plants was measured at changing $\mathrm{CO}_{2}$ concentrations, initially twice or three times higher than under natural conditions. The highest mean photosynthesis rate in laminae amounted* to $13-14 \mathrm{mg}$ $\mathrm{CO}_{2} \mathrm{dm}^{-2} \mathrm{~h}^{-1}$ at chlorophyll content $3-4 \mathrm{mg} \mathrm{dm}^{-2}$; it was lower than in the investigations on wheat** carried out by Stoy (1965) but higher than that reported by Larcher (1963).

In the here reported experiments, significant varietal differences in the photosynthesis rate in laminae (even when they did not differ in chlorophyll content) could be demonstrated.

The prevailing opinion that there are no marked differences between varieties (Stoy 1965), and even species of wheat (Bielikow et al. 1961) in their assimilation rate seems to be based mainly on results obtained with leaves of young plants or with relatively young leaves of older plants and perhaps also at high $\mathrm{N}$ levels; in these cases also in the reported experiments no marked differences could be found. However, in the period of major importance for organic matter accumulation in grain such differences may occur and, moreover, the extent of nitrogen influence on the photosynthesis rate can be different in dependence on the varietal traits. It should be added that there was no proportionality between chlorophyll content and photosynthesis rate even in older leaves, as has been reported by Sestak and Catsky to occur in Nicotiana (1962). The changes in the photosynthesis rate with ageing seem to depend mainly not on the chlorophyll content but on other processes possibly associated with changes in the photophosphorylation capacity (Smillie et al. 1959) or mitochondrial decomposition influencing chloroplast activity (Simon 1964) or else RNA synthesis etc. (Humphries et al. 1963). The rate of these processes can perhaps be different in various varieties, thus not only greater leaf area duration after heading but also longer maintainance of its high photosynthesis rate can be an important determinant of the high yielding capacity of the plant.

As previously mentioned, various authors directed their attention to ear photosynthesis. In our previous experiments a significant difference in this respect between Chłopicka and an awnless variety Fortunata has been demonstrated. The investigations reported showed that also awned varieties can differ significantly from each other in ear contribution.

It is worth stressing that the photosynthetic activity of the ear did not depend on the weight of its green parts, but rather on their chlorophyll content. Perhaps some varietal differences in the structure of glumes and awns are also involved (Grundbacher 1963). It is obvious that the share of the ear in the total shoot pholosynthetic activity depends to a high extent on the rate of senescence of the vegetative organs. However, the varietal differences in the contribution of the ear were significant not only at low, but also at high $\mathrm{N}$ level. The data obtained do

* Light intensity, initial and final $\mathrm{CO}_{2}$ concentration, as well as temperature during exposure to ${ }^{14} \mathrm{C}$ allowed to expect a higher rate of true/or very close to true/photosynthesis;

** Rate of photosynthesis of the flag leaf (with chlorophyll content $4 \mathrm{mg} \cdot \mathrm{dm}^{-2}$ ) at $70 \mathrm{Klux}$ accounted for about $30 \mathrm{mg} \mathrm{CO} \cdot \mathrm{dm}^{-2} \cdot \mathrm{h}^{-1}$. 
not allow to estimate the contribution of ear assimilates to grain yield. Thorne (1965) found that the amount of carbon expired by the wheat ear (including grain) was much higher than that assimilated by this organ. However, it should be borne in mind that the possible true contribution of ear photosynthates depends also on the differences as compared to shoot assimilates in the rate of their translocation to the grain and transformation to insoluble compounds, on the senescence rate of vegetative organs etc. It is worth stressing that a higher $\mathrm{N}$ supply had no influence on ear photosynthetic activity except of the rather late period of development and only in Chłopicka.

The influence of $\mathrm{N}$ applied at shooting seems to be of a complex character. In spite of a marked increase of the photosynthetic activity of the main shoot the amount of carbon assimilated before anthesis and found at ripeness was similar or only a little higher than that at low $\mathrm{N}$ level. The large differences in the assimilate loss from the aerial parts cannot have resulted mainly from differences in their respiration rate*. It seems that they were due partly to an incieased assimilate translocation to the root, and perhaps a better supply of this organ with carbohydrates at heading and anthesis allowed to maintain its activity for a longer time and similtaneously to delay senescence of the green plant parts**. Thus the photosynthates of lower leaves even, if they do not participate directly in carbon accumulation in the grain, may be of significance for its yield. Varietal differences as well as environment influence in this respect should be investigated.

There is no doubt that the greater assimilate loss from tillers at high $\mathrm{N}$ level is partly due to their higher number in comparison with that in the controls. Perhaps greater differences in assimilate loss in Popularna than Chłopicka resulted mainly from the greater influence of $\mathrm{N}$ on the tillering rate of the former.

It seems that the facts discussed above are of great importance when varietal differences in grain-yielding capacity are under consideration.

\section{SUMMARY}

Two awned wheat varieties Chłopicka and Popularna showed differences in favor of the latter in the dry weight of all plant parts and chlorophyll content. The greater leaf area in Popularna especially after heading was due mainly to the delayed senescence of lower leaves. The differences between the varieties in culm weight at ripeness were higher than at heading and greater than the differences in grain yield.

The higher $\mathrm{N}$ dose applied at shooting brought about an increase in chlorophyll content, leaf area duration and in grain yield.

The higher total photosynthetic activity of the shoots of Popularna before, but especially after heading, was due not only to the greater total green leaf area but also to a higher rate of photosynthesis per area unit, particularly in older leaves even when no differences in chlorophyll content occurred. The influence of $\mathrm{N}$ on the photosynthesis rate in these leaves was not always associated with its

* The differences in the size of the ear, culm and leaves caused by various $\mathrm{N}$ levels were very small at that time.

** The unpublished yet experimental data do not indicate that the great loss of carbon from the main shoot could have resulted from its translocation to fertile or sterile tillers. 
influence on chlorophyll changes, but rather with some other processes connected with senescence and influencing photosynthesis. In this respect the varieties investigated seem to differ from each other,

The photosynthetic activity of the green parts of the ear in Popularna - in spite of their higher weight - was lower or similar to that in the ear of Chłopicka. In most cases better $\mathrm{N}$ supply had no influence on ear photosynthesis.

The contribution of the particular green parts to the total photosynthetic activity of the shoots changed after heading greatly depending on the plant age, $\mathrm{N}$ supply and variety.

The efflux of assimilates from leaves to the culm is relatively rapid before and after anthesis and does not seem to be dependent on the "sink" capacity. The assimilates formed before anthesis (and even heading) and temporarily stored in the second and third (from the top) internodes are partially translocated to the grain at the stage of intensive filling.

The amount of photosynthates lost by the plants at high was greater than at low $\mathrm{N}$ level; so the differences in carbon accumulation at ripeness were relatively small, particularly when assimilates formed up to anthesis are concerned.

The amount of carbon assimilated a week before heading and found finally in grain ranged between 7 and $12 \%$ of its total at ripeness, being relatively lower in Popularna than in Chłopicka. The contribution of carbon assimilated between heading and anthesis gradually increased up to $40-50 \%$. Differences in $\mathrm{N}$ level had a very small influence or none on the final amount of carbon assimilated before anthesis in grain.

The contribution of assimilates formed during the first period of grain development increased to $75-80 \%$, reaching at the last stages $85-90 \%$ of the amount found at ripeness. The greater the influence of $\mathrm{N}$ on the photosynthetic efficiency of the plants after anthesis, the stronger is its influence on the final assimilate accumulation in the grain.

The role of lower leaves as well as of culm growth in the grain - yielding capacity of wheat varieties are discussed.

Plant Physiology Department

Agricultural University, Warsaw,

Plant Physiology Institute

Polish Academy of Sciences Warsaw

\section{REFERENCES}

Asana R. D., Saini A. D., 1958, Plant Phys. 11(4); 665.

Belikow P. S., Motorina M. W., Kurkowa E. B., 1961, Izw. Timir. Selsk.-Choz. Akad. 5:44. Birecka H., Dakić-Włodkowska L., 1963, Acta Soc. Pol. 32(4):631.

Birecka H., Dakić-Włodkowska L., 1964, ibidem 33(2):407.

Birecka H., 1966, Biul. Acad. Pol. Sci. ser. biol. 14(4):261

Bunting A. H., Drennan D. S. H., de Silva W. H. and Krishnamurthy K., 1964, Abstr. $10^{\text {th }}$ Inter. Bot. Congress 11 .

Buttrose M. S., 1963, Austr. I. Biol. Sci. 15(4):611.

Dobben v. W. H., 1962, Neth. J. Agr. Sci. 10:377.

Grundbacher F. J., 1963. Bot. Rev. 29(3):366

Hartt C. E., Kortschak H. P., Burr G. O., 1964, Pl. Phys. 39(1):15.

Hartt C. E., Kortschak H. P., 1964, ibidem, 39(3):460.

Humphries E. C., Weeler A. W., 1963, Ann. Rew. Pl. Phys. 14:385.

Larcher W., 1963, Mitc. Flor.-Soziol. Arbeitsgem. 10:120.

Lupton F. G. 1961, Ann. Appl. Biol. 49(3):557.

Nichiporowich A. A., Własowa M. K., 1961 Fizjol. Rast. (8) 1:19.

Nichiporowich A. A., 1961, Fizj. Rast. 8(5):536.

Nosberger J., Thorn e G. N., 1965, Ann. Bot. 29(116):635.

Silva W. H. de, 1961, (cf. Stoy W. 1965).

Simon E. W., 1964, Abstr. 10 $0^{\text {th }}$ Intern. Bot. Congress 198.

Smillie R. M., Krotkov G., 1959, Bioch. Bioph. Acta 33:550. 
Stoy V., 1965, Phys. Plant. Supp. IV.

Sestak Z., Čatsky J., 1962 Biol. Plant. Acad. Sci. Bohem., 4(2):131.

Thorne G. N., 1962, J. Agr. Sci. 58:89.

Thorne G. N., 1965, Ann. Bot. 39(115):317.

Wardlaw I. F., 1965, Austr. J. Biol. Sci., 1965, 18(2):269.

Watson D. J., 1956, The growth of leaves, Proc. Third Easter School in Ags., Butterworths,

London. p. 178.

Watson D. J., Thorne G. N., French S. A. W., 1963, Ann. Bot. 27(105):1.

Welbank P. J., French S. A. W., Witts K. J., 1966., Ann. Bot. 30(118):291.

\section{Aktywność i produktywność fotosyntezy w roślinach pszenicy jarej $w$ okresie przed $i$ po wykloszeniu}

\section{Streszczenie}

Dwie przebadane ościste odmiany pszenicy jarej, 'Chłopicka' i 'Popularna' wykazały istotne różnice - na korzyść drugiej, - w ciężarze wszystkich organów, jak i w zawartości chlorofilu. Większa ogólna powierzchnia zielonych liści w 'Popularnej', szczególnie po wykłoszeniu, była głównie wynikiem wolniejszego starzenia się liści niższych pięter. Różnice między odmianami w ciężarze źdźbła w fazie dojrzewania były większe niż w okresie kłoszenia i jednocześnie większe niż różnice w ciężarze ziarna.

Większa dawka azotu zastosowana w okresie strzelania w źdźbło spowodowała zwiększenie zawartości chlorofilu w liściach, opóźnienie ich obumierania oraz wzrost masy ziarna.

Większa ogólna aktywność fotosyntetyczna pędów 'Popularnej' - w porównaniu z 'Chłopicką'była wynikiem nie tylko większej powierzchni zielonych liści, ale również większej intensywności fotosyntezy w przeliczeniu na jednostkę powierzchni (w szczególności liści starszych) nawet w przypadku braku różnic w zawartości chlorofilu. Wpływ azotu na intensywność asymilacji w tych liściach nie zawsze był skorelowany z jego wpływem na zmiany zawartości chlorofilu, ale raczej z innymi procesami związanymi z procesem starzenia i oddziaływającymi na fotosyntezę. Wydaje się, że przebadane odmiany różnią się między sobą pod tym względem.

Aktywność fotosyntetyczna kłosa w 'Popularnej' - mimo większego jego ciężaru - była niższa lub równa aktywności kłosa 'Chłopickiej'. W większości przypadków lepsze zaopatrzenie w azot nie miało wpływu na fotosyntezę w kłosie.

Udział poszczególnych zielonych organów w ogólnej aktywności fotosyntetycznej pędów ulegał po wykłoszeniu znacznym zmianom w zależności od wieku roślin, dawki azotu i odmiany.

Odpływ asymilatów z liści do źdźbła jest stosunkowo szybki zarówno przed, jak i po zapłodnieniu i nie wydaje się być zależny od ,pojemności" akceptora, uwarunkowanej intensywnością jego wzrostu. Asymilaty powstałe przed zapłodnieniem (a nawet kłoszeniem) i czasowo zakumulowane w drugim i trzecim od wierzchołka międzywęźlu są częściowo przemieszczane do ziaren w fazie ich intensywnego wypelniania.

Wielkość strat asymilatów w roślinach na wyższym poziomie azotu była większa niż na niższym; stąd też różnice w ich akumulacji pod koniec wegetacji były stosunkowo małe, szczególnie jeśli chodzi o związki organiczne powstałe przed zapłodnieniem.

W fazie pełnej dojrzałości w ziarniakach stwierdzono $7-12 \%$ pozostałej w roślinach ilości węgla zasymilowanego przed kłoszeniem. Względna akumulacja w ziarniakach węgla zasymilowanego w okresie między kłoszeniem i zapłodnieniem stopniowo wzrastała do $40-50 \%$, a w przypadku asymilatów powstałych w późniejszych fazach dochodziła do $75-90 \%$.

Różnice w poziomie azotu nie wywołały żadnych różnic, albo tylko bardzo małe w stopniu nagromadzenia węgla zasymilowanego przed zapłodnieniem. Natomiast jeśli chodzi o asymilaty powstałe w późniejszych fazach rozwoju, wpływ azotu na ich akumulację w ziarnie był tym większy, im większy był wpływ tego składnika pokarmowego na efektywność fotosyntezy.

Rola liści niższych pięter jak i procesu wzrostu źdźbla w produktywności odmian pszenicy jarej jest dyskutowana. 